\title{
Systemic inhibition of PTPN22 augments anticancer immunity
}

\author{
Won Jin Ho, ${ }^{1}$ Sarah Croessmann, ${ }^{2}$ Jianping Lin, ${ }^{3}$ Zaw H. Phyo, ${ }^{1}$ Soren Charmsaz, ${ }^{1}$ Ludmila Danilova, ${ }^{1}$ Aditya A. Mohan, ${ }^{1}$ \\ Nicole E. Gross, ${ }^{1}$ Fangluo Chen, ${ }^{1}$ Jiajun Dong, ${ }^{3}$ Devesh Aggarwal, ${ }^{3}$ Yunpeng Bai, ${ }^{3}$ Janey Wang, ${ }^{4}$ Jing He, ${ }^{4}$ James M. Leatherman, ${ }^{1}$ \\ Mark Yarchoan, ${ }^{1}$ Todd D. Armstrong, ${ }^{1}$ Neeha Zaidi, ${ }^{1}$ Elana J. Fertig, ${ }^{1}$ Joshua C. Denny, ${ }^{2,4,5}$ Ben H. Park, ${ }^{2}$ Zhong-Yin Zhang, ${ }^{3}$ and \\ Elizabeth M. Jaffee ${ }^{1}$ \\ 'Sidney Kimmel Comprehensive Cancer Center at Johns Hopkins, Baltimore, Maryland, USA. ${ }^{2}$ Department of Medicine, Vanderbilt University Medical Center, Nashville, Tennessee, USA. ${ }^{3}$ Department of \\ Medicinal Chemistry and Molecular Pharmacology, Department of Chemistry, Center for Cancer Research and Institute for Drug Discovery, Purdue University, West Lafayette, Indiana, USA. ${ }^{4}$ Department of \\ Biomedical Informatics, Vanderbilt University Medical Center, Nashville, Tennessee, USA. ${ }^{5}$ All of Us Research Program and National Human Genome Research Institute, NIH, Bethesda, Maryland, USA.
}

\begin{abstract}
Both epidemiologic and cellular studies in the context of autoimmune diseases have established that protein tyrosine phosphatase nonreceptor type 22 (PTPN22) is a key regulator of T cell receptor (TCR) signaling. However, its mechanism of action in tumors and its translatability as a target for cancer immunotherapy have not been established. Here, we show that a germline variant of PTPN22, rs2476601, portended a lower likelihood of cancer in patients. PTPN22 expression was also associated with markers of immune regulation in multiple cancer types. In mice, lack of PTPN22 augmented antitumor activity with greater infiltration and activation of macrophages, natural killer (NK) cells, and T cells. Notably, we generated a small molecule inhibitor of PTPN22, named L-1, that phenocopied the antitumor effects seen in genotypic PTPN22 knockout. PTPN22 inhibition promoted activation of CD8 ${ }^{+}$T cells and macrophage subpopulations toward MHC-II-expressing M1-like phenotypes, both of which were necessary for successful antitumor efficacy. Increased PD-1/PD-L1 axis expression in the setting of PTPN22 inhibition could be further leveraged with PD-1 inhibition to augment antitumor effects. Similarly, cancer patients with the rs 2476601 variant responded significantly better to checkpoint inhibitor immunotherapy. Our findings suggest that PTPN22 is a druggable systemic target for cancer immunotherapy.
\end{abstract}

\section{Introduction}

Recent progress in cancer immunotherapy has revolutionized the management of several cancer types (1). Understanding the

Authorship note: $\mathrm{WJH}, \mathrm{SC}$, and JL are co-first authors.

Conflict of interest: WH, SC, JL, ZYZ, BHP, and EMJ are co-inventors on invention disclosures related to targeting PTPN22 to treat cancers (W02021007491A1), developing novel compounds against PTPN22, and/or utilizing rs2476601 as a predictive biomarker for cancer immunotherapy (disclosed). WH is a co-inventor on patents with potential for receiving royalties from Rodeo Therapeutics. MY reports receiving research grants from Incyte, Bristol-Myers Squibb, and Exelixis, and is a consultant for AstraZeneca, Eisai, Exelixis, and Genentech. EJF is a consultant for Champions Oncology. BHP had ownership interest and was a paid member of the scientific advisory board of Loxo Oncology and was a paid consultant for Foundation Medicine, Inc, Lilly, Casdin Capital, and Roche. He is currently a paid scientific advisory board member of Celcuity and a paid consultant for Jackson Laboratories and Pathovax. Under separate licensing agreements between Horizon Discovery, LTD and The Johns Hopkins University, BHP is entitled to a share of royalties received by the University on sales of products. The terms of this arrangement are being managed by the Johns Hopkins University in accordance with its conflict of interest policies. ZYZ is a co-founder and serves on the scientific advisory board of Tyligand Bioscience. EMJ reports receiving a commercial research grant from Bristol-Myers Squibb

Reference information: J Clin Invest. 2021;131(17):e146950. https://doi.org/10.1172/ JCl146950.

Submitted: December 21, 2020; Accepted: July 16, 2021; Published: September 1, 2021. Copyright: @ 2021, American Society for Clinical Investigation.and Aduro Biotech, and is a consultant/advisory board member for Lustgarten Foundation, Parker Institute for Cancer Immunotherapy, CStone, Dragonfly, Genocea, Achilles and Adaptive Biotechnologies, and co-founder of Abmeta Biotech. importance of T cells in anticancer immunity has provided the foundation for many therapeutic options, including checkpoint inhibition and adoptive $\mathrm{T}$ cell approaches, e.g., chimeric antigen receptor $\mathrm{T}(\mathrm{CAR} \mathrm{T})$ cell therapies (2-4). However, most patients either have a limited or no response to current immunotherapies, warranting the ongoing search for additional immunotherapy strategies.

The fundamental process within $\mathrm{T}$ cells that links antigen recognition to cellular output is the $\mathrm{T}$ cell receptor (TCR) signaling cascade. Protein tyrosine phosphatase nonreceptor type 22 (PTPN22), a member of the PTP superfamily that is largely specific to immune cells, is a physiologic regulator of TCR signaling (5, 6). By dephosphorylating the activating tyrosine residues of Lck (Y394) and Zap70 (Y493), which are proximal kinases of the TCR signaling cascade, PTPN22 is able to subdue the activity of TCR signaling (7). In fact, numerous epidemiologic studies have established the association between a germline variant of PTPN22, C1858T (R620W; rs2476601) and autoimmune diseases (8-10). Accordingly, mouse studies have demonstrated that PTPN22 R619W (homologous to human R620W) is consistent with the loss-of-function phenotype seen in PTPN22-knockout (PTPN22KO) mice $(11,12)$. Furthermore, studies have shown that adoptive transfer of T cells lacking PTPN22 $(13,14)$, genetic KO of Ptpn22 in mice (15), and the presence of PTPN22 R619W in mice (16) all yield enhanced antitumor immune responses. These findings provide the impetus for establishing PTPN22 as a translatable target for cancer immunotherapy. 


\section{A}

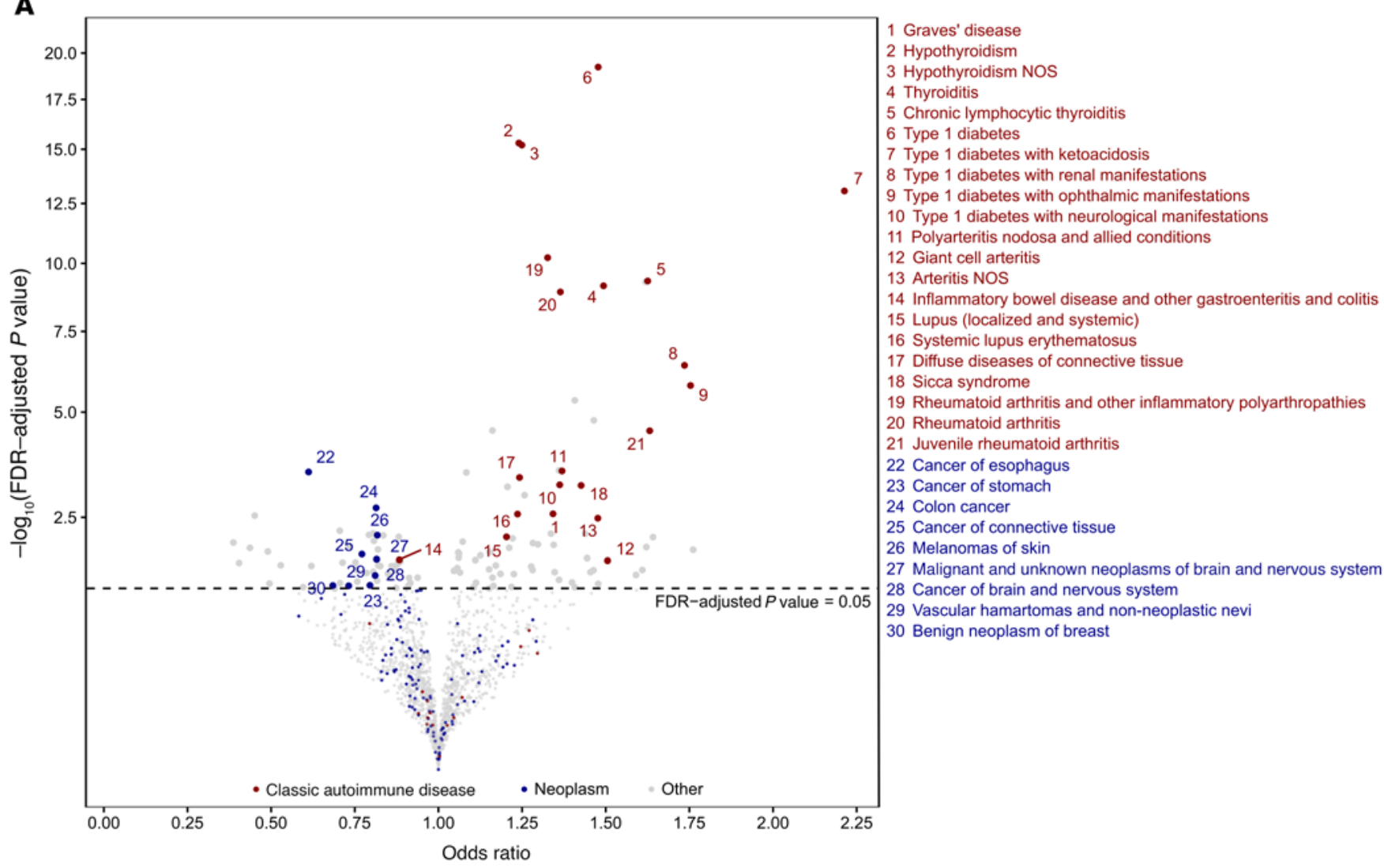

B

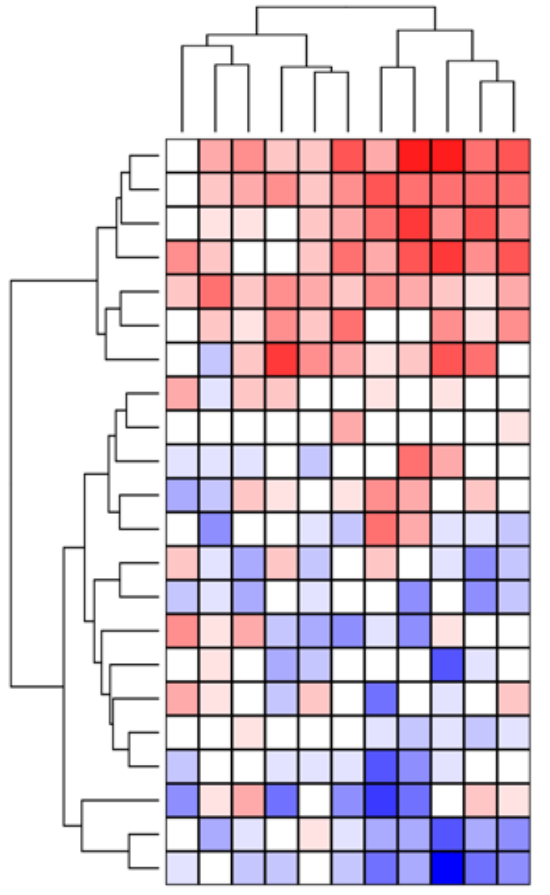

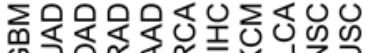

Macrophages.M1

T.cells.regulatory. Tregs.

T.cells.CD8

T.cells.CD4.memory.activated

Dendritic.cells.resting

T.cells.CD4.memory.resting

B.cells.naive

Neutrophils

T.cells.gamma.delta

NK.cells.activated

T.cells.follicular.helper

Plasma.cells

Mast.cells.activated

Macrophages.MO

Macrophages.M2

B.cells.memory

Monocytes

Eosinophils

NK.cells.resting

Mast.cells.resting

Dendritic.cells.activated

T.cells.CD4.naive

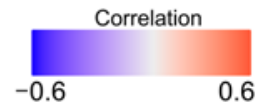

C

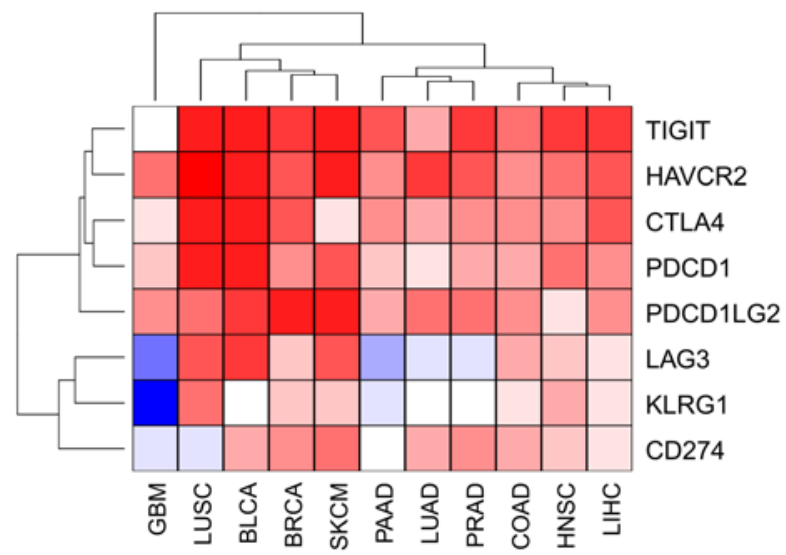


Figure 1. PTPN22 is associated with a negative regulatory role in the immune response against cancer. (A) A volcano plot showing the results from the analysis using phenome-wide association studies (PheWAS) from the Vanderbilt BioVU database $(n=72,083)$. Each dot represents an association between Ptpn22 rs 2476601 and a disease diagnosis. The horizontal dashed line indicates an FDR-adjusted $P$ value of 0.05 . (B) Correlations between Ptpn22 expression and immune cell types deconvolved by CIBERSORT across 11 cancer types from The Cancer Genome Atlas (TCGA) are shown as a heatmap. (C) Correlation between Ptpn22 and immune regulatory markers across 11 cancer types from TCGA are shown as a heatmap. BLCA, bladder cancer; BRCA, breast cancer; COAD, colon adenocarcinoma; GBM, glioblastoma multiforme; HNSC, head and neck squamous cell cancer; LIHC, hepatocellular carcinoma; LUAD, lung adenocarcinoma; LUSC, lung squamous cell carcinoma; PAAD, pancreatic ductal adenocarcinoma; PRAD, prostate adenocarcinoma; SKCM, skin melanoma.

Herein, we show that individuals with the PTPN22 rs2476601 variant are at increased risk for autoimmune disease and also have a reduced incidence of cancers. Using in vivo models, we show that genetic ablation of PTPN22 leads to augmented antitumor immune responses. We also designed and synthesized a small molecule inhibitor of PTPN22, named L-1, and show that pharmacologic inhibition of PTPN22 reproduces this phenotype. Furthermore, we demonstrate that both genetic and pharmacologic abrogation of PTPN22 enhances response to PD-1 inhibition. Finally, cancer patients with the rs2476601 variant have significantly greater responses to checkpoint inhibitor immunotherapy. Mechanistically, inhibition of PTPN22 with L-1 confers both antigen-specific T cell- and macrophage-mediated antitumor effects. Our results establish a molecular target that is druggable and may enhance responses to current immunotherapies.

\section{Results}

PTPN22 is associated with immune regulation of cancers. To first examine the potential role of PTPN22 in cancer development, we employed a large phenome-wide association study (PheWAS) database that explores relationships between the germline variant PTPN22 rs2476601 and human disease phenotypes (17). Based on genotyped DNA biobanks and deidentified electronic health records from Vanderbilt University Medical Center's BioVU, we found that the minor allele frequencies for rs2476601 were approximately $10 \%$ and $1.6 \%$ in the European $(n=72,083)$ and African $(n=$ $14,414)$ ancestry populations, respectively. In the European ancestry population, we were able to recapitulate the previously reported risk-conferring associations between PTPN22 rs2476601 and classic autoimmune diseases, namely rheumatoid arthritis, systemic lupus erythematosus, and thyroid disorders (Figure 1A). Importantly, a striking risk-preventive association between PTPN22 rs2476601 and multiple types of cancers was observed, with odds ratios mostly ranging from 0.6 to 0.8 for melanoma, gastrointestinal cancers, and central nervous system cancers. In the African ancestry population, the associations were present but less significant (Supplemental Figure 1A; supplemental material available online with this article; https://doi.org/10.1172/JCI146950DS1).

To gain additional insight into the functional relevance of PTPN22 in cancers, we used The Cancer Genome Atlas (TCGA) database to assess the correlations between PTPN22 expression and cell-type signatures across 11 cancer types. Using xCell
(18), a gene set-based deconvolution algorithm providing scores for over 65 cell types, we found that across 7 major cancer types examined, PTPN22 expression strongly correlated with scores for immune cell types and weakly correlated with scores for most other cell types (Supplemental Figure 1B). We then used a leukocyte-focused deconvolution algorithm based on linear support vector regression, CIBERSORT (19), to correlate immune cell subsets with PTPN22 expression. This analysis revealed that PTPN22 expression most highly correlated with $\mathrm{T}$ cell and inflammatory (M1) macrophage subsets, especially in melanoma (Figure 1B). In inflamed cancer types, PTPN22 expression also correlated strongly with the expression of other markers of immune regulation (CD274, PDCD1, CTLA4, LAG3, HAVCR2; Figure 1C). When stratifying by level of expression (top $20 \%$ vs. lower $80 \%$ ), univariate survival analysis results were similar to those of other key immune markers, e.g., CD4, CD8A, PDCD1, and CTLA4 (Supplemental Figure 1C). Expression of PTPN22 was weakly correlated (0.142) with overall tumor mutational burden (Supplemental Figure 1D). Together, these results suggested that PTPN22 is involved in the negative regulation of anticancer immunity.

Lack of PTPN22 augments anticancer immunity. Building on these observations, we directly interrogated the role of PTPN22 in anticancer immune responses using PTPN22-KO mice (12). First, MC38 carcinoma cells in the C57BL/6J background were subcutaneously injected into wild-type (WT) or PTPN22-KO C57BL/6J mice to compare tumor growth in syngeneic immunocompetent mice (Figure 2A). As expected, tumor growth, gross tumor sizes, and tumor weights were significantly lower in PTPN22-KO mice compared with WT mice (Figure 2, B and C). Characterizing the tumors by immunohistochemistry demonstrated a significantly increased presence of $\mathrm{CD}^{+}$and $\mathrm{CD}^{+} \mathrm{T}$ cells within the tumors without relative increases in Foxp3 $3^{+} \mathrm{T}$ cells (Figure 2, D and E). Global immune profiling of the tumors was performed using cytometry by time-of-flight (CyTOF) (20) and analyzed by FlowSOM (21) and UMAP (22) algorithms, which identified a total of 16 immune cell subtypes (Supplemental Figure 2A). Many cell types, including $\mathrm{T}$ cells, tumor-associated macrophage (TAM) subsets, and NK cells were increased in the PTPN22-KO tumors (Figure $2 \mathrm{~F}$ ). A more focused analysis of the same CyTOF data set on 9 subtypes within the $\mathrm{T}$ cell population (Figure $2 \mathrm{G}$ ) showed that among the subtypes that are increased, cytotoxic $\mathrm{CD} 8^{+} \mathrm{T}$ cells expressing granzyme B were increased to the greatest extent in the tumors from PTPN22-KO versus WT mice. Further CyTOF profiling revealed that PTPN22-KO tumors were infiltrated with a greater abundance of IFN- $\gamma$-expressing cytotoxic T cell subsets and IL-2expressing helper $\mathrm{T}$ cell subsets (Supplemental Figure 2C). Significant increases in both $\mathrm{CD}^{+}$and $\mathrm{CD}^{+} \mathrm{T}$ cells within the tumors were further confirmed by flow cytometry (Figure $2 \mathrm{H}$ ).

PTPN22 is a systemically druggable immunotherapy target. To extend the translatability of these findings, we sought to test whether systemic inhibition of PTPN22 with a pharmacological agent is feasible and leads to a phenotype comparable to the genetic KO. To identify PTPN22 inhibitors with the requisite pharmacological properties, we screened a small collection of drug-like molecules for PTPN22 inhibitory activity. We discovered a quinolone derivative (Core 1, Supplemental Figure 3A) that inhibits PTPN22 with an $\mathrm{IC}_{50}$ of $432 \pm 45 \mu \mathrm{M}$. Through a structure-guided and fragment-based 
A

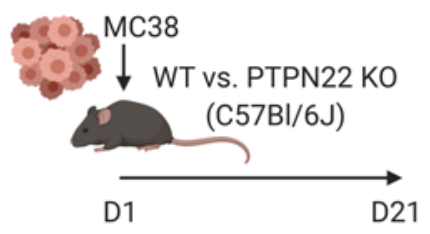

D

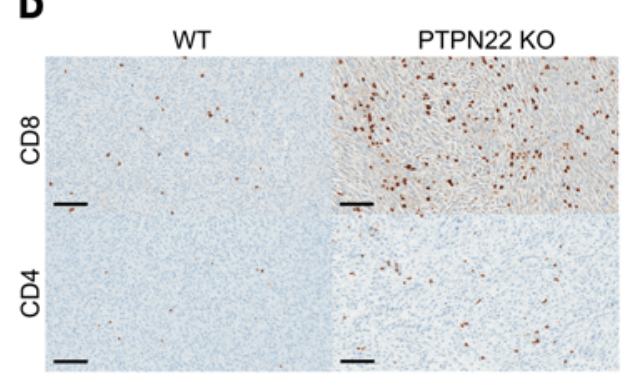

$\mathbf{F}$
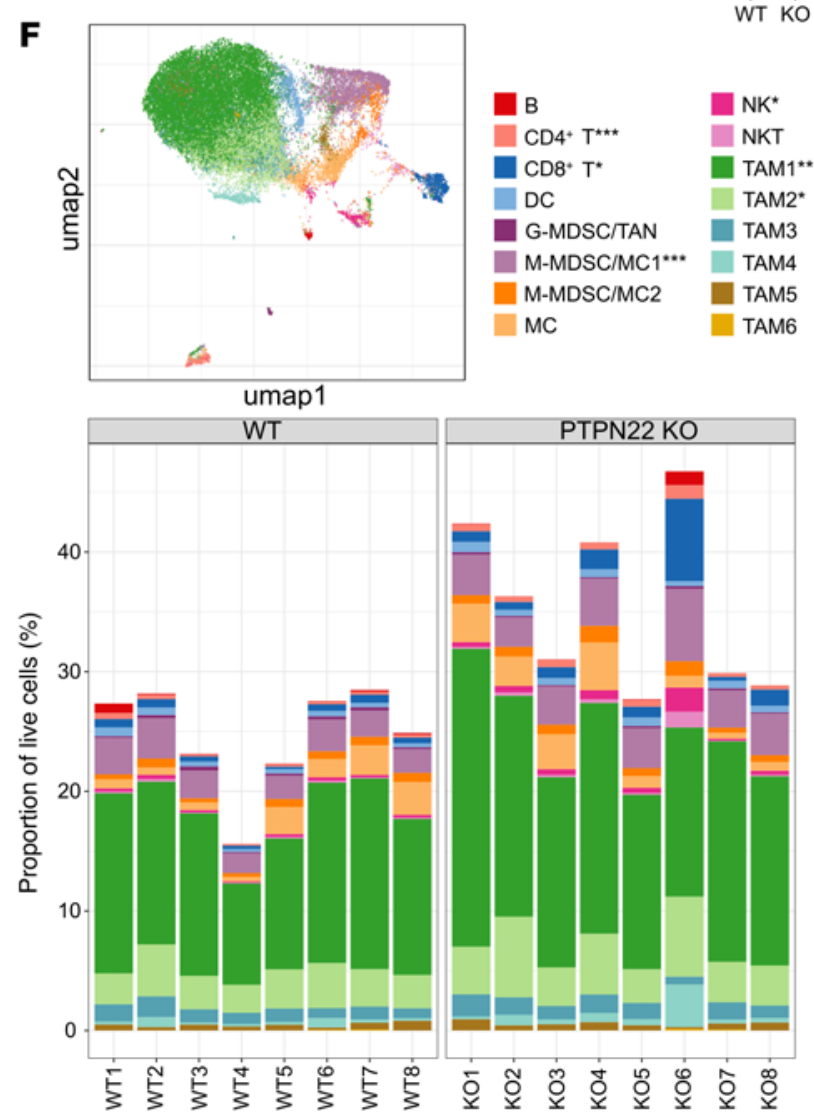
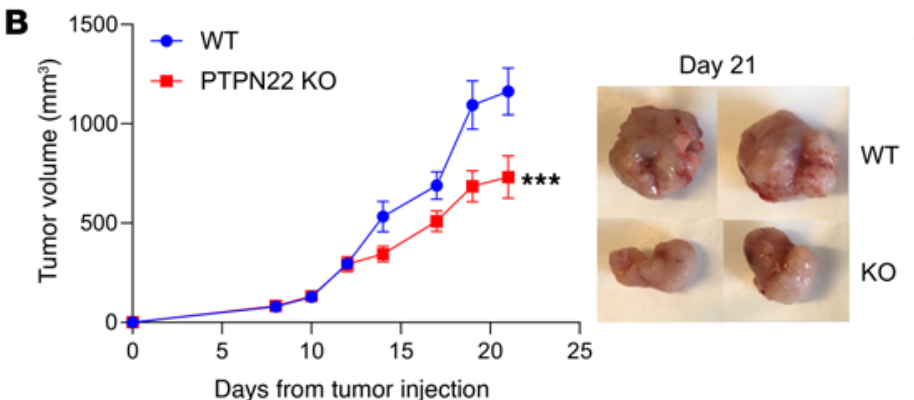

C

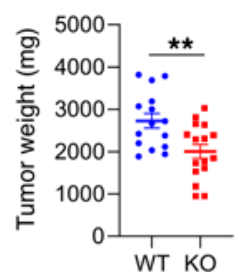

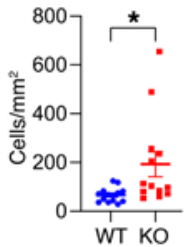

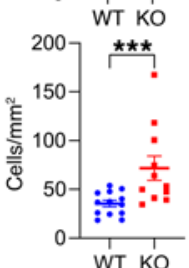

苍
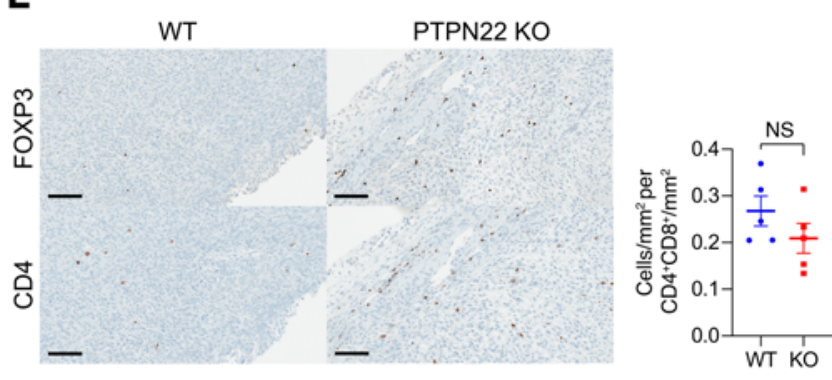

\section{G}

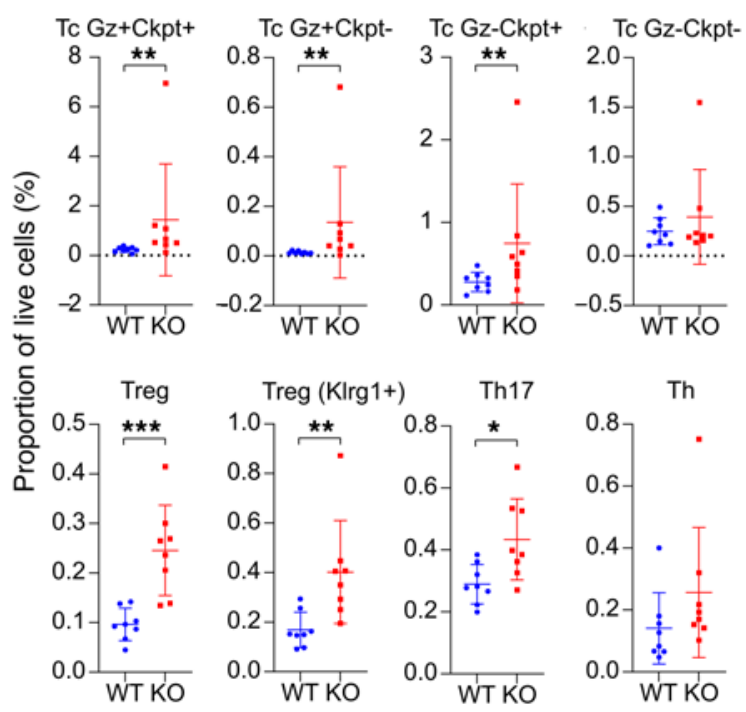

H

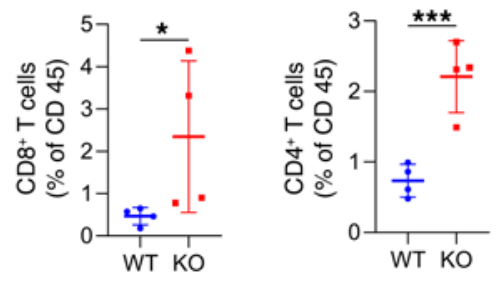


Figure 2. PTPN22 KO confers protection against MC38 tumor growth in association with enhanced immune infiltration. (A) Schematic of the mouse tumor model used. Cells $\left(2.5 \times 10^{5}\right)$ were injected subcutaneously in the right hind limb for tumor growth measurements 2 to 3 times a week through 21 days after injection. (B) MC38 tumor growth was compared between WT (blue circles) and PTPN22-KO (red squares) mice ( $n=10-11$ per arm). ${ }^{* *} P<0.001$ by nonlinear regression. Pictures on the adjacent panel show gross morphology of the 2 representative tumors from each arm. (C) Weights of the MC38 tumors on the day of the harvest (day 21) from WT and PTPN22-KO mice ( $n=15-16)$. ( $\mathbf{D}$ and E) Immunohistochemical analysis of the tumors comparing $\mathrm{CD} 4^{+}$and $\mathrm{CD} 8^{+}$cells and $\mathrm{Foxp}^{+}$cells infiltrating the tumors (scale bars: $100 \mu \mathrm{m}$ ). Positive staining was quantified using HALO software ( $n=11-13$ per arm). Foxp3 $3^{+}$density is represented as a proportion of $\mathrm{T}$ cell density (sum of $\mathrm{CD} 4^{+}$and $\mathrm{CD} 8^{+}$densities). ${ }^{*} P<0.05,{ }^{* * *} P<$ 0.005 by 2 -tailed, unpaired $t$ test. (F and $\mathbf{G}$ ) Immune subsets and T cell subsets are represented as percentage of live cells (WT vs. PTPN22-KO, mean \pm SEM, $n=8)$. ${ }^{*} P<0.05,{ }^{* *} P<0.01,{ }^{* *} P<0.005$. UMAP plot from CyTOF analysis of the immune profile is shown. Detailed annotations of cell types are shown in Supplemental Figure 2A. (H) Conventional flow cytometry was performed to validate CyTOF findings showing increased CD8 ${ }^{+} T$ cells and $\mathrm{CD}^{+} \mathrm{T}$ cells within the MC38 tumors in PTPN22-KO mice compared with WT $(n=4) .{ }^{*} P<0.05,{ }^{* * *} P<0.005$ by 1 -tailed, unpaired $t$ test. Ckpt, checkpoint markers; DC, dendritic cells; G-MDSC/TAN, granulocytic myeloid-derived suppressor cells/tumor associated neutrophils; Gz, granzyme; M-MDSC/MC, monocytic myeloid-derived suppressor cells/myeloid cells; MC, monocytes; NK, natural killer cells; NKT, natural killer T cells; TAM, tumor-associated macrophages; Tc, cytotoxic T cells; Th, helper T cells; Treg, regulatory T cells.

focused library approach, compound L-1 consisting of Core 1 with an L-alanine linker and a biphenyl carboxylic group as the tail fragment, was identified as the most potent inhibitor of PTPN22 with an $\mathrm{IC}_{50}$ value of $1.4 \pm 0.2 \mu \mathrm{M}$, which represented nearly a 310-fold gain in binding affinity compared with the parent Core 1 . Selectivity profiling revealed that L-1 exhibits greater than 7- to 10-fold selectivity for PTPN22 over 16 similar PTPs (Supplemental Figure 3B). Further kinetic analyses revealed that $\mathrm{L}-1$ is a competitive inhibitor for PTPN22 with a $K_{\mathrm{i}}$ value of $0.50 \pm 0.03 \mu \mathrm{M}$ (Supplemental Figure $3 \mathrm{C})$. Since L-1 is fairly hydrophobic, we also developed a formulation utilizing an emulsifier, cremophor-EL, to improve its in vivo pharmacokinetic properties. Administration of L-1 intraperitoneally at $10 \mathrm{mg} / \mathrm{kg}$ yielded an average AUC of $4.55 \mu \mathrm{M} \cdot \mathrm{h}$ and $\mathrm{C}_{\max }$ of 1.11 $\mu \mathrm{M}$ (Supplemental Figure 3D), which is more than twice its $K_{\mathrm{i}}$ value.

As observed in the PTPN22-KO model, treatment of WT mice with L-1 (Figure 3A) led to significantly reduced MC38 tumor growth compared with the vehicle-injected control group (Figure 3B). We further tested the effect of L-1 on another syngeneic immunocompetent model of different genetic background, CT26 in BALB/c mice, which showed similar antitumor effects (Figure 3C). Deep profiling of both MC38 (Supplemental Figure 4, A-C) and CT26 (Supplemental Figure 4D) tumor immune infiltrates using CyTOF showed a significantly enhanced presence of TAM and $\mathrm{CD}^{+}$and $\mathrm{CD}^{+} \mathrm{T}$ cell subtypes in L-1-treated tumors. Functional marker profiles within each of the immune cell subtypes also were comparable between the PTPN22-KO and L-1-treated conditions in the MC38 tumor model (Supplemental Figure 5A). In both cases, systemic abrogation of PTPN22 generally led to higher expression of CD69, PD-1, and LAG3 in T cells; CD40, CD69, and PD-L1 in the TAM subsets; and CD69 and granzyme B in the NK cells, indicating enhanced activation in these cell types. Similar changes were again observed in the CT26 model (Supplemental Figure 5B). Analysis of the MC38 tumors with immunohistochemistry visually confirmed that L-1 induced increased infiltration, with significant and near-significant numbers of $\mathrm{CD}^{+}(P=0.049)$ and $\mathrm{CD} 4^{+} \mathrm{T}$ cells $(P=0.056)$, respectively (Figure 3D). To test whether the effects of $\mathrm{L}-1$ could be attributable to off-target effects, we treated MC38 tumors in WT and PTPN22-KO mice with either vehicle or L-1 injections. No significant differences in tumor growth were noted between vehicle- and L-1-treated PTPN22-KO mice, suggesting that the L-1-mediated protective effects against tumor growth were PTPN22 and host specific (Figure 3E). Collectively, these findings showed that PTPN22 is a druggable target for immunotherapy.

Antitumor efficacy of PTPN22 abrogation is dependent on T cells. To understand whether the protective effects incurred by PTPN22 abrogation are indeed dependent on T cells, we compared the tumor growth in PTPN22-KO mice upon $\mathrm{CD}^{+}$or $\mathrm{CD} 8^{+} \mathrm{T}$ cell depletion. As predicted, in the presence of depleting anti-CD8 antibodies, tumor growth was significantly accelerated (Figure $4 \mathrm{~A}$ ). $\mathrm{CD}^{+} \mathrm{T}$ cell depletion only mildly increased tumor growth rates. We also performed TCR sequencing and found that there was reduced TCR diversity in tumor-infiltrating T cells (Figure 4B) in line with a higher degree of clonality (Figure 4C) when PTPN22 is genetically or pharmacologically antagonized. Together, these results suggested that the antitumor T cell response enhanced by inhibition of PTPN22 is an antigen-driven process that leads to productive clonal T cell expansion. However, since the TCR repertoire does not indicate whether these clones are truly tumor specific, we tested PTPN22 loss in another tumor model with a defined antigen, the lymphoma cell line EL4-OVA injected subcutaneously into syngeneic C57BL/6J mice. Since EL4-OVA tumor growth appeared to be more variable, the rate of rejection was assessed instead. By day 35 from the day of tumor injection, PTPN22-KO mice had nearly double the rate of tumor rejection compared with WT mice (9/20 vs. 5/20; Figure 4D). The OVA-secreting nature of the tumor was then exploited to determine whether the antitumor immune response is tumor specific. Tetramer analysis demonstrated a trend in which PTPN22-KO mice had the highest number of SIINFEKL-specific tetramer-positive $\mathrm{CD}^{+} \mathrm{T}$ cells within the tumor-draining lymph nodes (Figure $4 \mathrm{E}$ ). Since naive antigen-specific $\mathrm{T}$ cell responses against the EL4-OVA model are known to be limited without prior vaccination $(23,24)$, we then compared the degree of antigen-specific responses seen in the PTPN22-KO versus WT mice using a peptide vaccination strategy. Indeed, when WT and PTPN22-KO mice were vaccinated with SIINFEKL peptides, PTPN22-KO mice generated significantly greater numbers of antigen-specific $\mathrm{CD}^{+} \mathrm{T}$ cells (Figure $4 \mathrm{~F}$ ).

Phosphorylation of PTPN22-specific phospho-sites corresponds with markers of activation in $C D 8^{+} T$ cells. Since the infiltration of granzyme $\mathrm{B}^{+} \mathrm{CD}^{+} \mathrm{T}$ cells into tumors was most prominently enhanced in the PTPN22-KO mice, and PTPN22 is responsible for removing activating tyrosine phosphorylation in Lck and Zap70, we hypothesized that the phosphorylation states of PTPN22-specific tyrosine residues, Lck Y394 and/or Zap70 Y493, may correlate with markers of activation within $\mathrm{CD}^{+} \mathrm{T}$ cells. To determine the states of key phosphorylation sites along the TCR signaling cascade simultaneously with multiple lineage and functional markers at the single-cell level, we developed and validated a CyTOF panel for phospho-immune profiling of $\mathrm{CD}^{+} \mathrm{T}$ cells (Supplemental Figure 6, A-C). Using this CyTOF panel, we first 
A

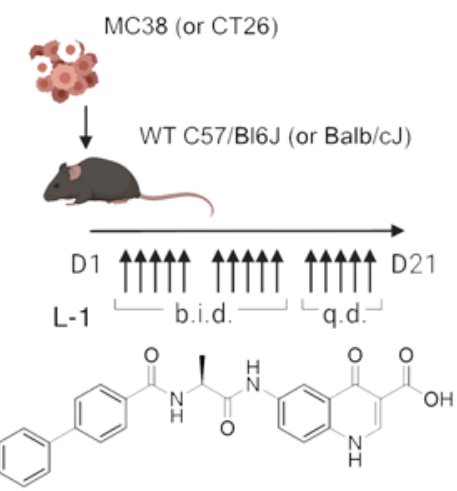

C

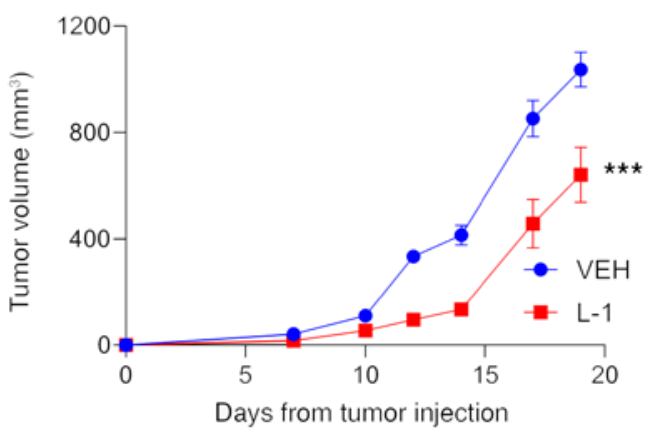

E

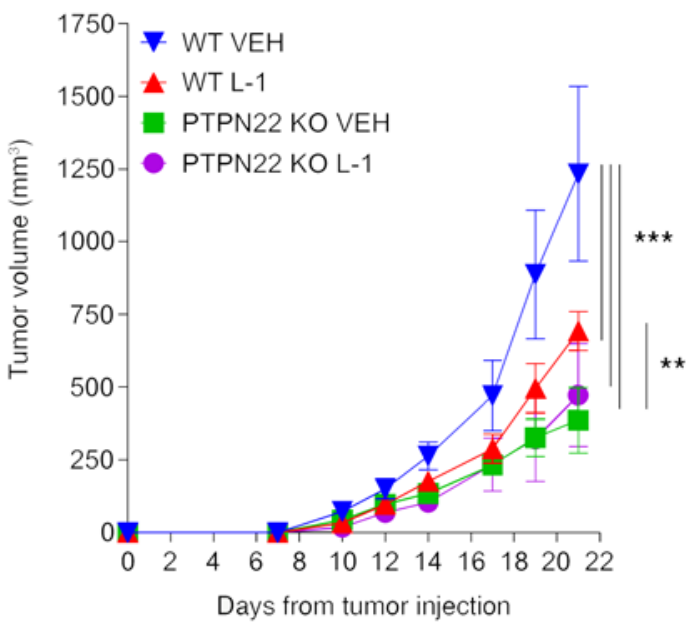

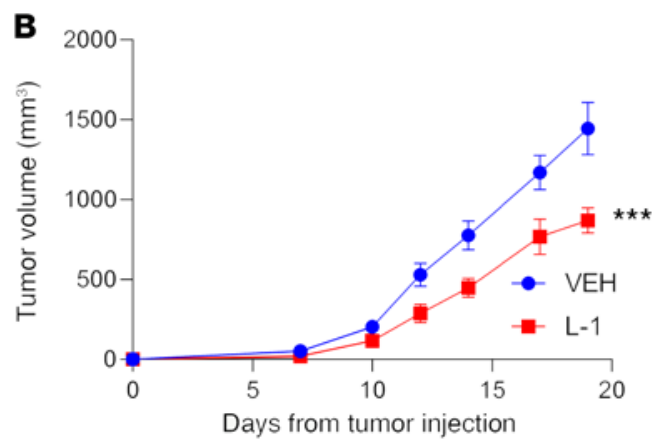

D
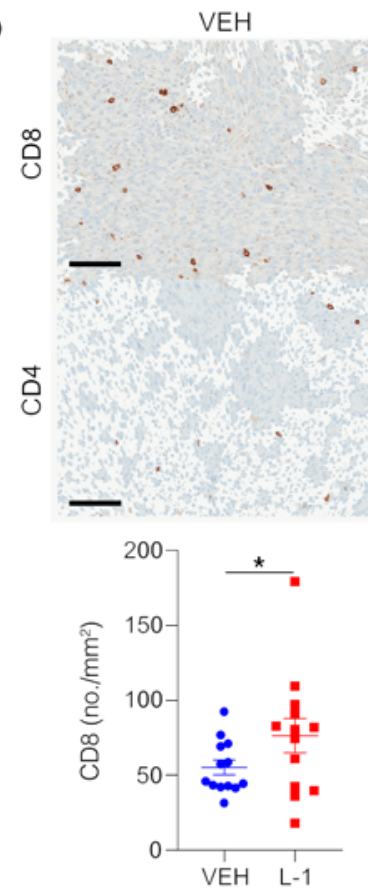

Figure 3. Treatment with a small molecule inhibitor of PTPN22, L-1, phenocopies PTPN22 KO. (A) Schematic of the L-1 treatment of MC38 and CT26 tumor model. Starting on day 3 of injection, L-1 is administered intraperitoneally twice daily for 5 consecutive days per week for 2 weeks and once daily for 5 consecutive days per week for 1 week. The structure of L-1 is illustrated. (B and C) Tumor growth of MC38 in C57BL/6) and CT26 in BALB/C) was compared between vehicle (blue circles) and L-1 (red squares) treatment groups. Mean \pm SEM ( $n=9-10$ per arm). ${ }^{* * *} P<0.001$ by nonlinear regression. (D) Immunohistochemical analysis of the MC38 tumors shows CD4+ and CD8 ${ }^{+}$cells infiltrating the tumors (scale bars: $100 \mu$ m). Positive staining was quantified using HALO software, and the results are shown as mean \pm SEM ( $n=10-13$ per arm). ${ }^{*} P<0.05$ by 1 -tailed, unpaired $t$ test. (E) To assess potential off-target effects of L-1, starting on day 3 of injection, WT or PTPN22-KO mice were given either vehicle (VEH) or L-1 intraperitoneally. Tumor growth curves for WT VEH (blue inverted triangles), WT L-1 (red triangles), PTPN22-KO VEH (green squares), and PTPN22-KO L-1 (purple circles). Mean \pm SEM ( $n=4-5$ per arm). ${ }^{* *} P<0.01 ;{ }^{* *} P<0.005$ by nonlinear regression; not significant between PTPN22-KO VEH and PTPN22-KO L-1.

phospho-profiled CD8 ${ }^{+} \mathrm{T}$ cells from MC38 tumor-bearing mouse spleens. The spleen was selected to explore correlations among the markers assayed since there would be wider dynamic ranges. In the resulting correlation matrix (Supplemental Figure 6D), we first observed that among the TCR signaling phospho-sites, Zap70 Y493, Lck Y394, LAT Y226, MEK1 S298, and p38 MAPK Y182 were highly correlated; and CD247 Y142, Lck Y505, and
ERK Y204 were less correlated. Among the functional markers, strongest correlations were noted between granzyme B and Ki67, and between the checkpoint markers. Of all phospho-sites, Zap70 Y493, LAT Y226, MEK1 S298, and p38 MAPK Y182 correlated most strongly with the activation markers. We then sought to verify these correlations using an independent set of samples. Since many of the antibody clones were generated specifically against 
human epitopes, we phospho-profiled $\mathrm{CD}^{+} \mathrm{T}$ cells to obtain a comparable correlation matrix using deidentified peripheral blood samples from patients with hepatocellular carcinoma naive to immunotherapy (Supplemental Figure 6E). Overall, correlations were generally weaker in the human data set. However, the most appreciable correlations were among Zap70 Y493, Lck Y394, LAT Y226, and p38 MAPK Y182, similar to what was observed in mice. The highest correlation between phospho-sites was seen between the two PTPN22-specific sites Zap70 Y493 and Lck Y394. Among the functional markers, correlation between granzyme B and Ki67 was again the strongest. Furthermore, consistent with the mouse data set, Zap70 Y493, Lck Y394, and p38 MAPK Y182 were highly correlated with granzyme B and Ki67 among all phospho-sites. These findings together support a direct link between activation of tumor-killing $\mathrm{CD}^{+} \mathrm{T}$ cells and activation of phosphorylated PTPN22-specific sites downstream of both the classical (i.e., Zap70/LAT/MEK1) and the alternative pathways (i.e., Zap70/p38 MAPK; refs. 25, 26), for TCR signal transduction.

Enhanced TCR phospho-profiles in tumor-infiltrating $C D 8^{+} T$ cells associate with activated and exhausted phenotypes. Next, to understand (a) whether the changes in the TCR signaling phospho-profiles in the $\mathrm{CD}^{+} \mathrm{T}$ cells within the tumor microenvironment are in fact different between WT and PTPN22-KO, and (b) whether these changes are specific to a particular functional state of $\mathrm{CD} 8^{+} \mathrm{T}$ cells, we phospho-profiled tumor-infiltrating leukocytes from MC38 tumors using CyTOF. We clustered the data set into 16 differentiation states of $\mathrm{CD}^{+} \mathrm{T}$ cells (Supplemental Figure 6F) and discovered that the phosphorylation levels among all assayed TCR signaling phospho-sites increased in coordination with memory and exhausted (the expression of checkpoint markers) phenotypes (Figure 4G). When comparing between WT and PTPN22$\mathrm{KO}$, the most significant difference among all phospho-sites was the PTPN22-specific Lck Y394. Higher levels of phosphorylation in PTPN22-KO were noted early in the $\mathrm{CD}^{+} \mathrm{T}$ cell programming, being detected in early activated and central memory subtypes. These results suggested that the lack of PTPN22 and thus, increased activity of Lck, leads to enhanced TCR signal transduction and $\mathrm{T}$ cell activation. This in turn may foster augmented effector $\mathrm{T}$ cell functions and differentiation toward memory and exhaustion states upon antigen recognition. This interpretation is consistent with the significantly increased infiltration of granzyme $\mathrm{B}^{+} \mathrm{T}$ cells and checkpoint marker-expressing $\mathrm{T}$ cells in the MC38 tumors from PTPN22-KO and L-1-treated mice (Figure 2G and Supplemental Figure 4C). To further confirm that L-1 leads to PTPN22-specific phosphorylation changes, we treated mice for 3 days with L-1, as well as a structurally related but inactive compound L-17 (i.e., L-1 lacking the carboxylate group on the quinolone core, $\mathrm{IC}_{50}$ for PTPN22> $100 \mu \mathrm{M}$; see Supplemental Methods), via subcutaneous pumps and performed phospho-flow cytometry on splenocytes fixed immediately upon harvest, given the short half-life of L-1. Indeed, CD8 ${ }^{+} \mathrm{T}$ cells exhibited higher phosphorylation levels at Lck Y394 and Zap70 Y493 upon treatment with L-1, but not L-17 (Supplemental Figure 6G).

The state of $\mathrm{T}$ cell exhaustion is a nuanced characterization. Expression of checkpoint markers occurs downstream of $\mathrm{T}$ cell activation and does not necessarily indicate terminal exhaustion $(27,28)$. To determine whether the lack of PTPN22 promotes terminal exhaustion, we assayed for the transcriptional factors Tbet (a marker of nonterminal exhaustion) and eomesodermin (EOMES, a marker more indicative of terminal exhaustion), in the tumor-infiltrating $\mathrm{CD}^{+} \mathrm{T}$ cells $(27,28)$. Interestingly, while the proportion of $\mathrm{Tbet}^{\mathrm{hi}} \mathrm{CD} 8^{+} \mathrm{T}$ cells was higher in the MC38 tumors from PTPN22-KO than WT mice, the proportions of $\mathrm{EOMES}^{+}$and EOMES ${ }^{+}$Tbet $^{\mathrm{hi}} \mathrm{CD}^{+}{ }^{+} \mathrm{T}$ cells were not significantly different (Supplemental Figure 7A). This was observed also in L-1-treated CT26 tumors (Supplemental Figure 7B).

Efficacy of PTPN22 inhibition is also mediated by TAM subpopulations. Since PTPN22 inhibition enhanced the activation of TAMs in addition to T cells, we sought to discern whether they are necessary contributors to L-1 efficacy. Given that TAM populations were represented by F4/80 expression, we tested the effect of $L-1$ with or without an anti-F4/80 antibody. Interestingly, treatment of MC38 tumors with anti-F4/80 diminished the efficacy of L-1 (Figure $5 \mathrm{~A}$ ). This was in the setting of approximately $30 \%$ to $40 \%$ reduction of $\mathrm{F} 4 / 80^{+}$cells infiltrating tumors (Figure $5 \mathrm{~B}$ ). We then profiled the tumors with a CyTOF panel of antibodies that included markers to better phenotype macrophages (MHC-II [IA/IE], CD80, CD86 to define M1-like phenotype, i.e., proinflammatory; CD206 to define M2-like phenotype, i.e., antiinflammatory; ref. 29). Consistent with their known phenotypic significance, the expression of MHCII, CD80, and CD86 strongly correlated with each other, whereas CD206 was inversely correlated with those markers (Figure 5C). The resulting TAM clusters were then annotated into subpopulations of M1-like and M2-like TAMs based on their expression profiles (Figure 5D). Most notably, among the TAM clusters, we found that $\mathrm{L}-1$ treatment significantly reduced the abundances of only the CD206 ${ }^{+}$M2-like clusters with low MHC-II, CD80, and CD86 expression ("M2_IV", "M2_V”, "M2_VI"; Figure 5E). Furthermore, these changes were associated with significantly higher presence of T cells. Surprisingly, the effect of L-1 on enhancing the infiltration of multiple $\mathrm{T}$ cell subpopulations was suppressed with the subtotal depletion of F4/80 even when M2_IV, M2_V, and M2_VI abundances were low. This suggests that the T cell-promoting effects of PTPN22 inhibition may also require $\mathrm{F} 4 / 80^{+}$TAM subpopulations that are not M2_IV, M2_V, or M2_VI.

To directly interrogate the regulatory role of PTPN22expressing TAMs on $\mathrm{T}$ cell function, we magnetically selected TAMs $\left(\mathrm{F} 4 / 80^{+}\right)$from OVA-expressing MC38 tumors either in WT or PTPN22-KO mice and cocultured them with $\mathrm{CD}^{+}$OT-I or $\mathrm{CD}^{+}{ }^{+} \mathrm{OT}-\mathrm{II} \mathrm{T}$ cells enriched from spleens from OT-I or OT-II mice, respectively, at 1:10 and 1:1 TAM/T cell ratios. The MC38OVA model was selected for facilitating $\mathrm{T}$ cell responses specific for the OVA-expressing tumor cells while preserving the underlying MC38-associated biology. After 72 hours of coculture with TAMs, compared with controls with T cells only, CD8 ${ }^{+}$OT-I T cells were generally suppressed in number and function (CD69, IL-2, IFN- $\gamma$, and granzyme B expression), and CD4 ${ }^{+}$OT-II T cells were similarly suppressed in number and function (CD69, IL-2, and IFN- $\gamma$ expression; Supplemental Figure 8). However, we found that the number of CD8 ${ }^{+} \mathrm{OT}$-I cells was significantly less suppressed when cultured with tumor-derived PTPN22-KO TAMs than with WT TAMs (Supplemental Figure 8A). There were also trends toward higher numbers of OT cells when cultured with PTPN22-KO TAMs. These were accompanied by trends toward higher proliferative states by CFSE 
A

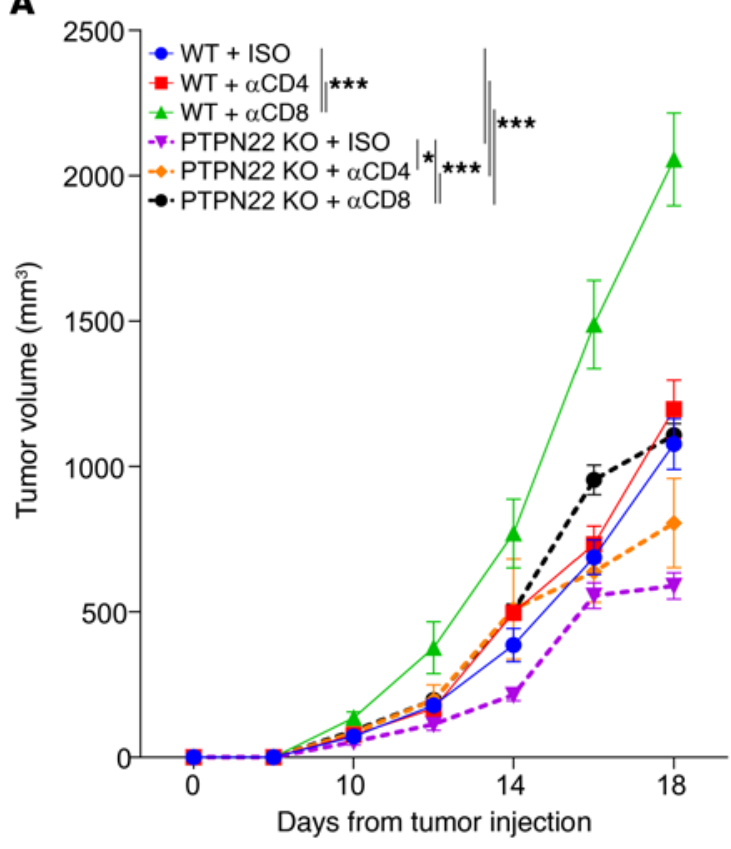

B

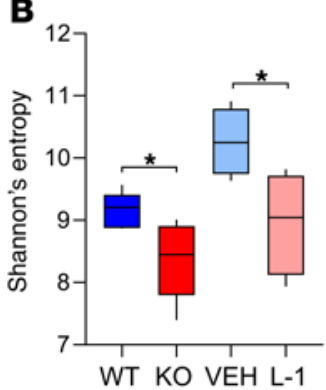

C

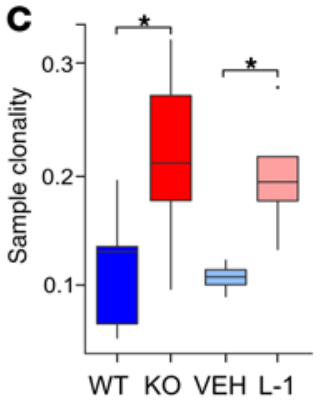

D

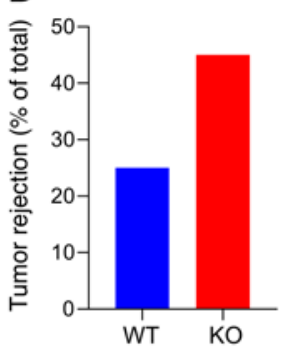

E

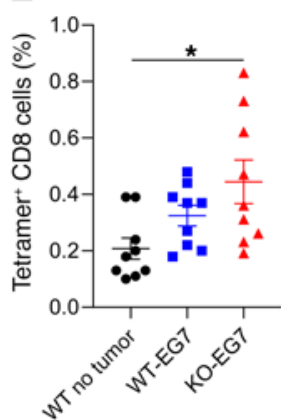

$\mathbf{F}$

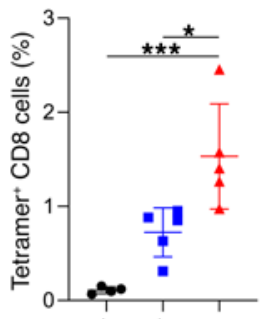

G
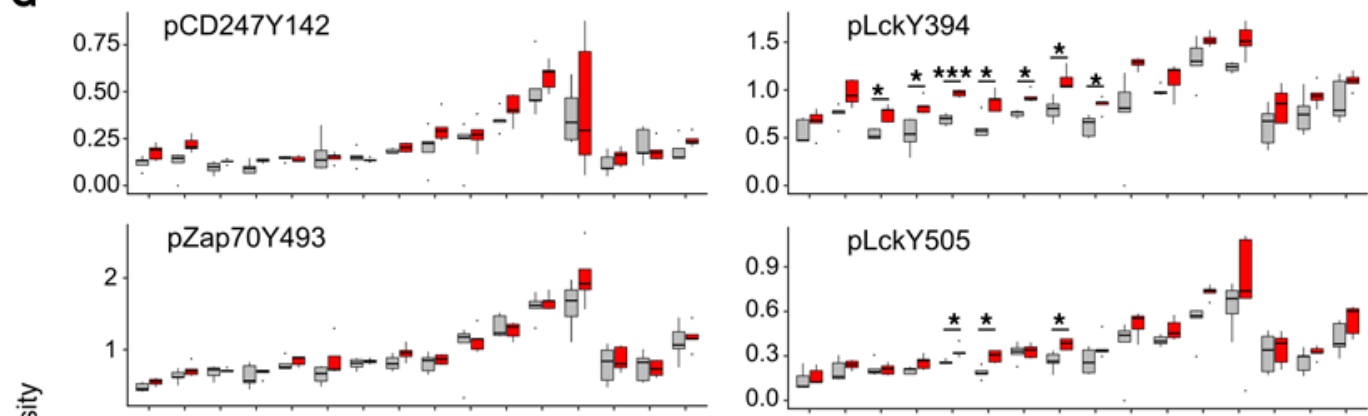

들
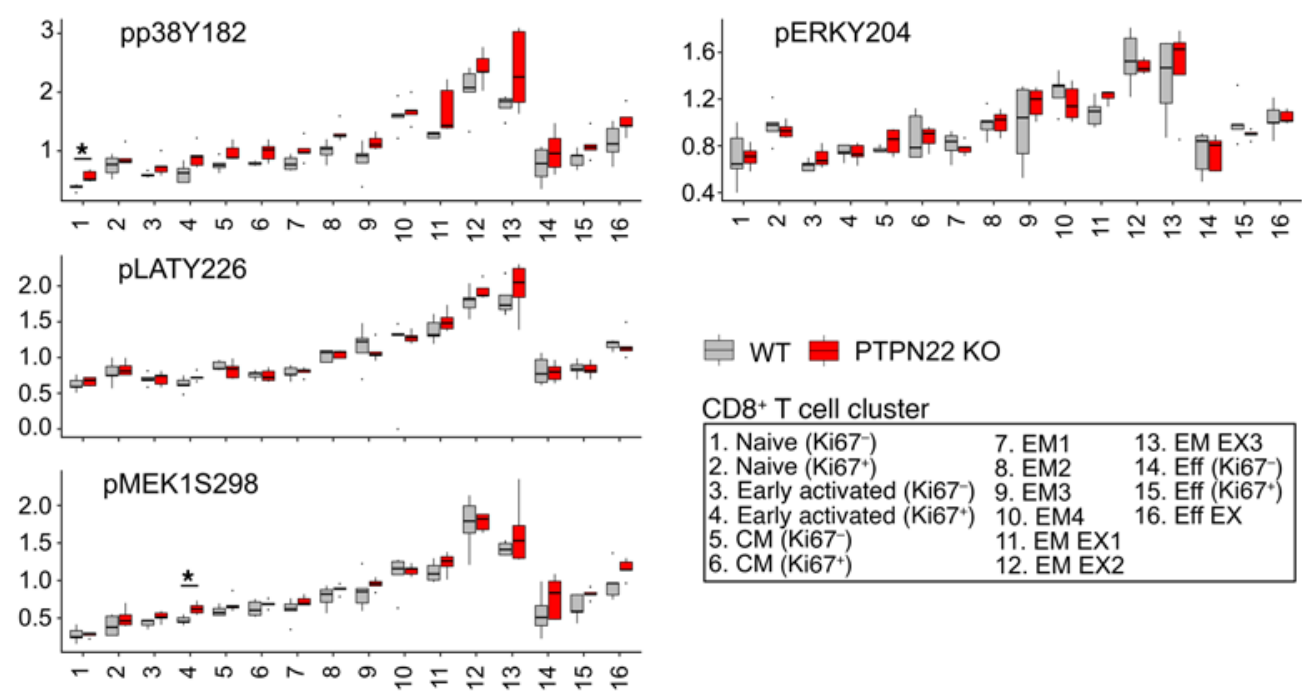

宙 $\mathrm{WT}$ PTPN22 KO

\begin{tabular}{|c|c|c|}
\hline $\begin{array}{l}\text { 1. Naive }\left(\mathrm{Ki} 67^{-}\right) \\
\text {2. Naive }\left(\mathrm{Ki} 67^{+}\right) \\
\text {3. Early activated }\left(\mathrm{Ki} 67^{-}\right) \\
\text {4. Early activated }\left(\mathrm{Ki} 67^{+}\right) \\
\text {5. CM }\left(\mathrm{Ki}^{-} 67^{-}\right) \\
\text {6. CM }\left(\mathrm{Ki} 67^{+}\right)\end{array}$ & $\begin{array}{l}\text { 7. EM1 } \\
\text { 8. EM2 } \\
\text { 9. EM3 } \\
\text { 10. EM4 } \\
\text { 11. EM EX1 } \\
\text { 12. EM EX2 }\end{array}$ & $\begin{array}{l}\text { 13. EM EX3 } \\
\text { 14. Eff }\left(\mathrm{Ki}^{-}\right) \\
\text {15. Eff }\left(\mathrm{Ki}^{+}\right) \\
\text {16. Eff EX }\end{array}$ \\
\hline
\end{tabular}


Figure 4. Antitumor effects of PTPN22 inhibition are mediated by CD8 ${ }^{+}$ T cells. (A) Tumor growth was compared across 6 groups: WT or PTPN22KO mice treated with isotype, anti-CD4, or anti-CD8 antibodies $(n=4-5)$. Representative of 2 independent runs. ${ }^{*} P<0.05,{ }^{* *} P<0.005$ by nonlinear regression. (B and $\mathbf{C}$ ) TCR repertoires for T cells infiltrating MC38 tumors were compared for WT vs. PTPN22-KO and VEH vs. L-1 treatments by TCRseq based on Shannon's entropy and sample clonality $(n=4-5)$. ${ }^{*} P<$ 0.05. (D) Tumor resistance experiment with EG7 tumors: $2.5 \times 10^{5}$ cells were injected subcutaneously in the right hind limb, and tumor persistence was assessed on day 35. The frequency of tumors rejected in WT and PTPN22$\mathrm{KO}$ mice is displayed $(n=20)$. (E) SIINFEKL tetramer ${ }^{+} \mathrm{CD} 8^{+} \mathrm{T}$ cells in the tumor-draining lymph nodes from EG7 tumor-bearing mice were compared by flow cytometry using 1-way ANOVA followed by pairwise Tukey's test $(n=9) .{ }^{*} P<0.05$. (F) SIINFEKL tetramer+ $C D 8{ }^{+} T$ cells from the spleens of vaccinated mice $(n=5)$. ${ }^{*} P<0.05,{ }^{* *} P<0.005$ by 1 -way ANOVA followed by pairwise Tukey's test. (G) Phosphorylation intensities (mean metal intensities) for each of the indicated phospho-site, stratified by the subtype of CD8 ${ }^{+} T$ cells, comparing MC38 tumor-infiltrating CD8 ${ }^{+} T$ cells from WT and PTPN22-KO mice $(n=5)$. In the box-and-whisker plots in B, C, and G, the bottom and top hinges of the boxes mark the 25th and 75th percentiles, respectively, and the lines within the boxes are medians. Whiskers represent 1.5 times the interquartile range extending from the hinges. Results of linear mixed modeling for differential analyses of the phosphorylation levels are shown as FDR-adjusted $P$ values: ${ }^{*} P<0.1$, ${ }^{* *} P<0.005$. Detailed annotations of $\mathrm{CD}^{+} \mathrm{T}$ cell clusters are shown in Supplemental Figure 7. CM, central memory subtype; EM, effector memory subtype; Eff, effector subtype; EX, exhausted subtype (positive expression of checkpoint markers).

assay. Most notably, IFN- $\gamma$ expression was significantly higher in CD8 ${ }^{+}$OT-I T cells when cultured with a higher number of TAMs and even more so when cultured with PTPN22-KO TAMs (Supplemental Figure 8B). In CD4 ${ }^{+}$OT-II T cells, CD69 expression was also significantly higher when cultured with more TAMs and PTPN22-KO TAMs when compared with WT TAMs (Supplemental Figure 8C). These results further support our findings that PTPN22 abrogation reprograms TAMs to be less suppressive of cancer-specific T cells and importantly, enhances $\mathrm{T}$ cell function and activation depending on the T cell subset (IFN- $\gamma$ in $\mathrm{CD}^{+}$and CD69 in $\mathrm{CD}^{+}$).

Furthermore, to understand how $\mathrm{T}$ cells may influence TAM populations, we performed CyTOF profiling of tumors from the $\mathrm{T}$ cell depletion study (in the cohort shown on Figure 4A). Consistent with the results from other experiments, MC38 tumors in PTPN22KO mice exhibited a significant increase in M1-like TAMs and reduction in M2-like TAMs compared with WT (Supplemental Figure 9A). Upon $\mathrm{CD}^{+} \mathrm{T}$ cell depletion, however, this effect was significantly suppressed. Interestingly, $\mathrm{CD} 4^{+}$depletion, which eliminated both helper $\mathrm{T}$ cells and regulatory $\mathrm{T}$ cells, was associated with enrichment of M1-like TAMs. Taken together, these data support a positive feedback loop between $\mathrm{CD}^{+} \mathrm{T}$ cells and M1-like TAMs to enhance their antitumor function.

Finally, since NK cells were also enriched upon PTPN22 abrogation, we evaluated NK cell depletion in L-1-treated mice. However, L-1 efficacy was not reduced following NK cell depletion using an anti-NK1.1 antibody (Supplemental Figure 9B).

Targeting PTPN22 is nonredundant with and augments antiPD-1 therapy. Since PTPN22 abrogation leads to higher expression of PD-1 and Tbet in $\mathrm{CD}^{+} \mathrm{T}$ cells along with higher expression of PD-L1 in TAMs, we hypothesized that checkpoint inhibition would reinvigorate the $\mathrm{Tbet}^{\mathrm{hi}} \mathrm{CD} 8^{+} \mathrm{T}$ cells and act against the PD-1/PD-L1 axis in the tumor microenvironment (30) to further enhance the tumor-resistant phenotype of PTPN22-KO mice. Indeed, when mice were treated with anti-PD-1 therapy, the tumor-resistant effects seen in the PTPN22-KO mice was augmented significantly (Figure 6A). Furthermore, when PD-1 inhibition was tested in combination with L-1 and compared against either L-1 only or antiPD-1 only, the combination arm was superior to any of the monotherapy arms in both MC38 and CT26 models (Figure 6B). Finally, we explored the potential clinical impact of PTPN22 in anticancer immunity by comparing the responses to checkpoint immunotherapy in cancer patients with or without the PTPN22 rs2476601 variant as a case-control study, comprised of similar patient characteristics in both groups (Table 1). Compared with matched control patients, a significant enhancing effect of checkpoint inhibitor immunotherapy was seen in patients with the PTPN22 rs2476601 variant (Figure 6) without significant differences in immune-related adverse events (Table 2). Interestingly, patients with the greatest responses to checkpoint immunotherapy in the control group had received multiple checkpoint inhibitors, whereas comparable responses could be observed in the setting of single checkpoint inhibition for patients in the variant group (annotated in Figure 6).

\section{Discussion}

Our study establishes 2 key concepts: (a) small molecule inhibition of PTPN22 is a feasible approach to improving cancer immunotherapy, the demonstration of which is critical for realizing the potential of PTPN22 as a target for cancer immunotherapy, and that (b) systemic inhibition of PTPN22 exerts antitumor immune effects by modulating T cells and in concert with TAMs. PTPN22 was previously shown to have $\mathrm{T}$ cell regulatory function. Consistent with our findings, adoptive cell therapy studies demonstrated that $\mathrm{CD} 8^{+} \mathrm{T}$ cells lacking PTPN22 are superior in clearing established tumors $(13,14)$ via multiple mechanisms, including the enhanced capacity to overcome TGF- $\beta$-mediated immunosuppression by increased IL-2 production as well as improving effector and memory functions. During this study period, another group also published $\mathrm{T}$ cell-dependent findings in syngeneic tumors that are consistent with ours in the context of genetic abrogation of PTPN22 (15). The use of catalytically inert Ptpn22227s/227s-knockin mouse models in that study further demonstrated that the loss of PTPN22 phosphatase activity is critical for enhancing antitumor responses. Functionally, we show that upon PTPN22 abrogation, T cells acquire higher phosphorylation levels at PTPN22-specific sites in association with increased memory states and expression of the proimmune cytokines IL-2 and IFN- $\gamma$, the cytolytic molecule granzyme $\mathrm{B}$, the activation marker CD69, and the checkpoint marker PD-1. While these findings indicate improved activation of $\mathrm{T}$ cells, the role of PD-1 is to provide negative regulatory feedback during activation (31). Our results have shown that the increased expression of PD-1 as regulatory feedback to pharmacologic PTPN22 inhibition, together with increased T-bet ${ }^{\text {hi }}$, which represents a non-terminally exhausted state, can be additionally exploited by anti-PD-1 therapy to yield even greater enhanced antitumor effects. These findings provide the rationale to expand the translational implications of targeting PTPN22 in cancer immunotherapy.

The role of PTPN22 in innate immune cells has remained less clear, but there is evidence that lack of PTPN22 promotes polarization of macrophages toward a proinflammatory M1 phenotype 


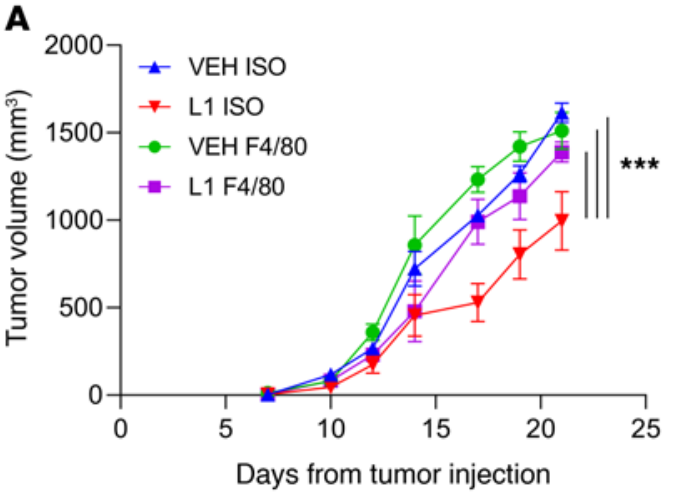

B

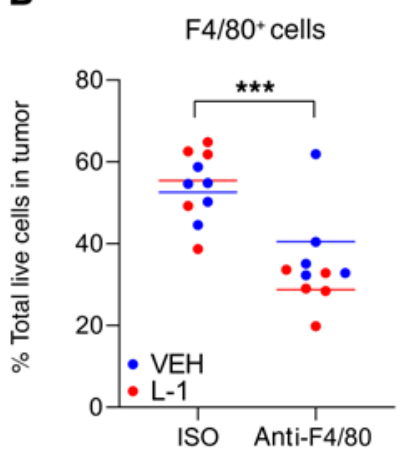

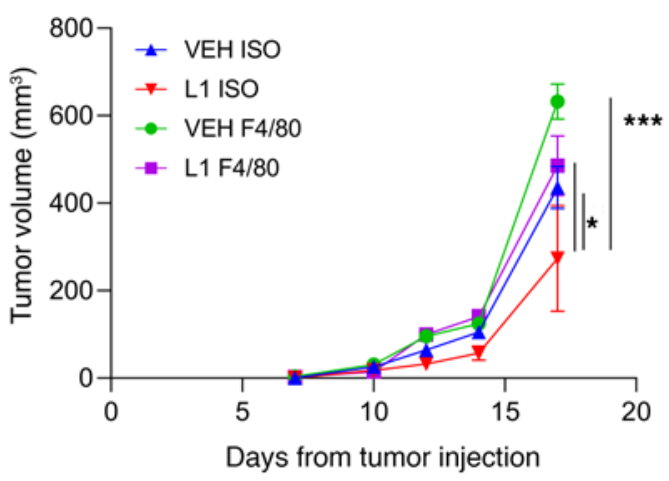

C

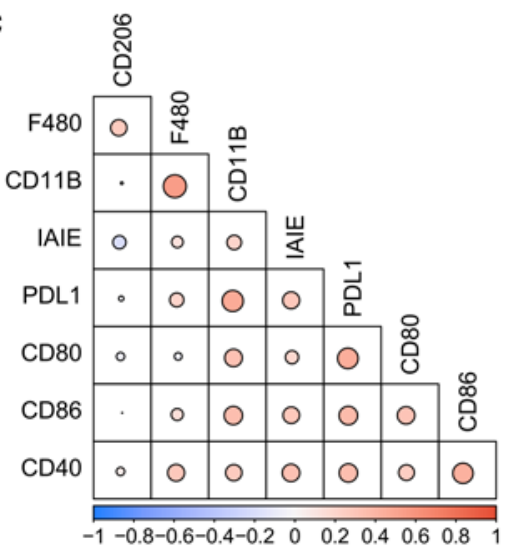

D

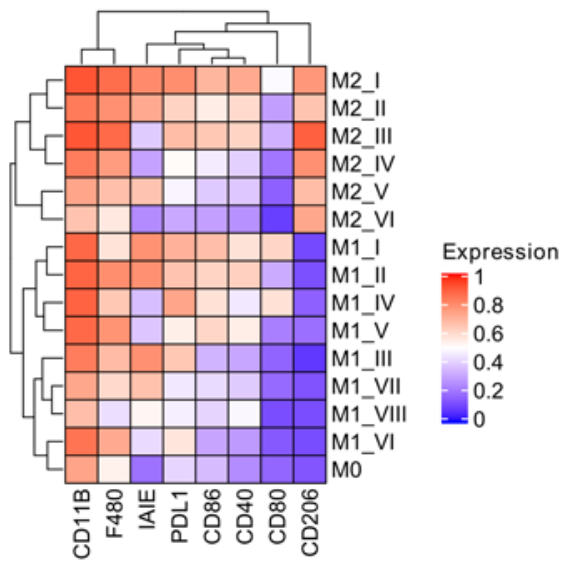

E

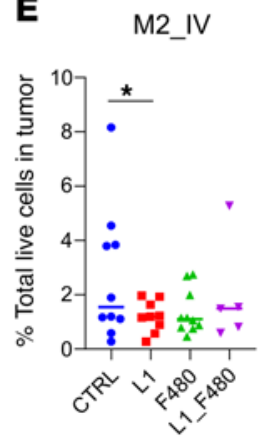

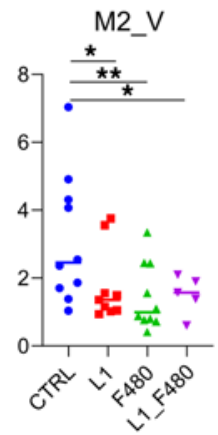
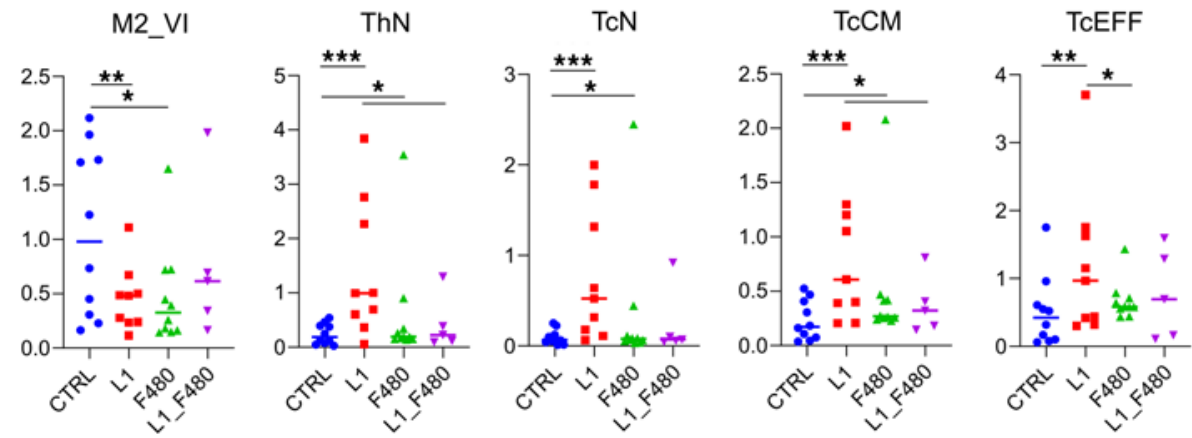

Figure 5. Remodeling of F4/80+ TAM compartment mediates the antitumor efficacy of L-1. (A) Two independent replicate runs testing the effects of VEH vs. L-1 with or without anti-F4/80 on MC38 tumor growth are shown ( $n=3-5$ for run 1 [left] and $n=5$ for run 2 [right]). ${ }^{*} P<0.05$, ${ }^{* *} P<0.005$ by nonlinear regression. (B-E) CyTOF analysis of the F4/80+ populations in the MC38 model. (B) The extent of anti-F4/80 depletion observed within the tumors. ${ }^{* * *} P<0.005$ only for the anti-F4/80 effect based on 2-way ANOVA. (C) Correlations among phenotypic markers relevant to TAMs within the TAM data subset. (D) Hierarchically clustered expression heatmap of annotated TAM clusters. (E) The proportion of TAM and T cell subpopulations within the tumors across the 4 groups $(n=5-10)$. ${ }^{*} P<0.05$, ${ }^{* *} P<0.01,{ }^{* *} P<0.005$ by 1 -way ANOVA.

(32), increases monocytic secretion of proinflammatory cytokines (33), and increases CD40 expression in dendritic cells (leading to higher proliferation of cocultured $\mathrm{CD} 4^{+} \mathrm{T}$ cells; ref. 34). Our findings identify a mechanism by which PTPN22 inhibition reprograms TAMs to be more antitumor M1-like and specifically $\mathrm{T}$ cell enhancing, and shows a codependency with the presence of $\mathrm{CD}^{+} \mathrm{T}$ cells in the tumor microenvironment. One potential mediator may be $\mathrm{CD} 8^{+}$ T cell-driven IFN- $\gamma$, which was increased upon PTPN22 abrogation and coculture with PTPN22-KO TAMs, since IFN- $\gamma$ polarizes macrophages toward the M1-like phenotype, i.e., classical activation (35). Thus, our results further extend prior observations, and also show for the first time to our knowledge that PTPN22 inhibition can exert antitumor immune effects through TAMs and their interactions with $\mathrm{T}$ cells. This is particularly provocative, given that systemic inhibition of PTPN22 would simultaneously reap the benefits of enhancing $\mathrm{T}$ cell function and reprogramming the TAM compartment to be less immunosuppressive, whereas other $\mathrm{T}$ cell-oriented approaches, e.g., CAR T cell therapies, would not alter TAMs.

PTPs have historically been considered poor targets for drug development, given the highly conserved and positively charged PTP active sites. However, accumulating evidence has increasingly challenged the notion that PTPs are not viable drug targets (36-38). 

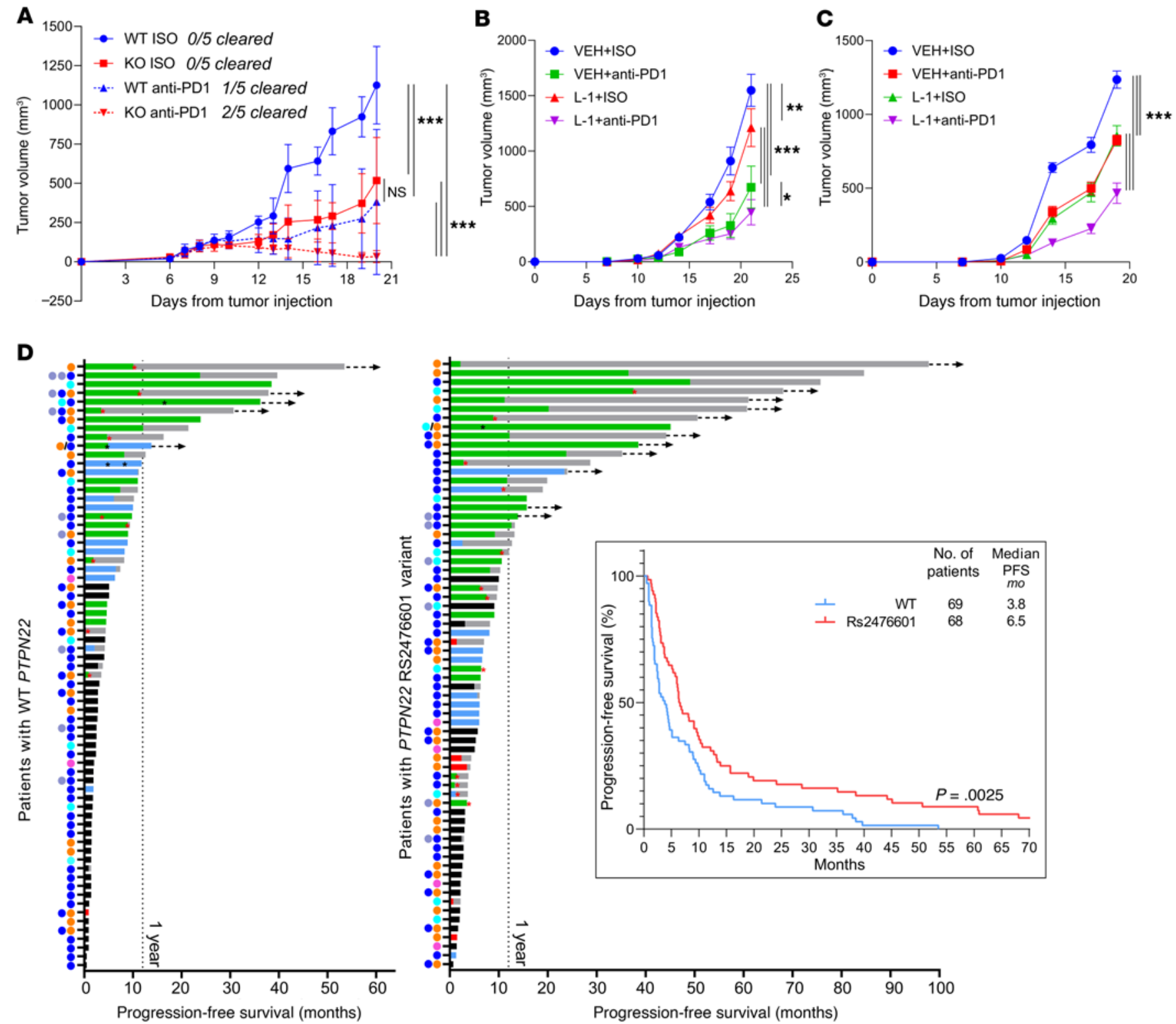

\begin{tabular}{|c|c|}
\hline $\begin{array}{l}\text { Time on therapy: Best response } \\
\text { PR,R } \\
\text { SD } \\
\text { PD * Small PD, pseudo PD } \\
\text { TOX * Stop RX for tox }\end{array}$ & $\begin{array}{l}\text { Treatment } \\
\text { Atezolizumab } \\
\text { Ipilimumab } \\
\text { Nivolumab } \\
\text { Pembrolizumab }\end{array}$ \\
\hline $\begin{array}{l}\text { Time w/o progression } \\
\ldots \rightarrow \text { PF as of last correspondence }\end{array}$ & Combination therapy \\
\hline
\end{tabular}

Figure 6. Targeting PTPN22 synergizes anti-PD-1 therapy. (A) Tumor growth experiment testing the effects of a single 200- $\mu$ g dose (day 8) of anti-PD-1 therapy against MC38 tumors in WT and PTPN22-KO mice. Growth curves are shown for 4 groups: WT or PTPN22-KO mice treated with isotype antibody (WT ISO or PTPN22 KO ISO) or anti-PD-1 antibody (WT anti-PD-1 or PTPN22 anti-PD-1) $(n=5)$. ${ }^{* *} P<0.005$ by nonlinear regression. (B and C) Combination of PTPN22 inhibitor L-1 with or without anti-PD-1 therapy $(n=9-10)$ in MC38 (B) or CT26 (C) models. ${ }^{*} P<0.05,{ }^{* *} P<0.01,{ }^{* * *} P<0.005$ by nonlinear regression. (D) Response to checkpoint immunotherapy in patients with rs2476601 or WT Ptpn22 from the BioVU database. "Combination Therapy" (gray dots) refers to a combination of the indicated immunotherapy with a nonimmunotherapeutic drug. Inset: Kaplan-Meier curves comparing progression-free survival.

Our results provide a proof of concept that PTPN22 can be targeted both specifically and with robust in vivo biological activity using a small molecule inhibitor. By using L-1, which specifically targets the active site of PTPN22, our study further bolsters the previously observed importance of PTPN22 phosphatase activity in regulating antitumor immune responses (15). Based on our findings, we propose that successful development of small molecule inhibitors of PTPN22 would provide a novel class of immunotherapies and excite new combination strategies to be explored.

With regard to the relatively common rs 2476601 variant of PTPN22, an important study has previously demonstrated that the variant leads to the degradation and decreased expression of PTPN22 in lymphocytes as an underlying mechanism for their hyperresponsiveness (34). This interpretation is in line with our findings that 


\section{Table 1. Patient demographics}

\begin{tabular}{|c|c|c|c|}
\hline Characteristic & $\begin{array}{c}\text { All patients } \\
(n=137)\end{array}$ & $\begin{array}{c}\text { rs2476601 SNP } \\
(n=68)\end{array}$ & $\begin{array}{c}\text { Control group } \\
(n=69)\end{array}$ \\
\hline \multicolumn{4}{|l|}{ Age at treatment, yr } \\
\hline Mean \pm SD & $62.6 \pm 11.0$ & $62.1 \pm 12.1$ & $63.5 \pm 9.1$ \\
\hline Median (range) & $64(31-86)$ & $63(31-86)$ & $65(33-80)$ \\
\hline \multicolumn{4}{|l|}{ Sex, no. (\%) } \\
\hline Female & $47(34)$ & $31(46)$ & $16(23)$ \\
\hline Male & $90(66)$ & $37(54)$ & $53(77)$ \\
\hline \multicolumn{4}{|c|}{ Race or ethnic group, no. (\%) } \\
\hline White & 131 (96) & 67 (99) & 64 (93) \\
\hline Black & $3(2)$ & 0 & $3(4)$ \\
\hline Unknown & $3(2)$ & $1(1)$ & $2(3)$ \\
\hline \multicolumn{4}{|c|}{ Primary diagnosis, no. (\%) } \\
\hline HNSCC & $9(7)$ & $4(7)$ & $5(7)$ \\
\hline Melanoma & $42(31)$ & $21(31)$ & $21(31)$ \\
\hline NSCLC & $37(27)$ & $19(28)$ & $18(26)$ \\
\hline SCLC & $9(7)$ & $3(4)$ & $6(9)$ \\
\hline $\mathrm{RCC}$ & $17(12)$ & $7(10)$ & $10(14)$ \\
\hline Urothelial & $8(6)$ & $6(9)$ & $2(3)$ \\
\hline Other & $15(11)$ & $8^{A}(10)$ & $7(10)$ \\
\hline
\end{tabular}

AThree of the 8 patients categorized as "other" contained 2 primaries. All 3 patients had lung tumors as at least one of their primaries. Two of the 4 patients contained 2 lung primaries. HNSCC, head and neck squamous cell carcinoma; NSCLC, non-small cell lung cancer; SCLC, small cell lung cancer; RCC, renal cell carcinoma. authentication by STR DNA profiling was done at the Johns Hopkins Genetic Resources Core Facility. All cell lines were tested for mycoplasma and were negative.

Animal models. WT C57BL/6J, OT-I (C57BL/6-Tg(TcraTcrb)1100Mjb/J), OT-II (B6.Cg-Tg(TcraTcrb)425Cbn/J), BALB/ cJ, and PTPN22-KO (B6.Cg-Ptpn22tm2Achn/J) mice were purchased from The Jackson Laboratory and bred in house. For syngeneic immunocompetent mouse models, age-matched 8- to 12-week-old female mice were injected with MC38 (2.5 $\times$ $\left.10^{5}\right)$ or EL4-OVA $\left(2.5 \times 10^{5}\right.$ for rejection and $5 \times 10^{5}$ for treatment) or CT26 $\left(5 \times 10^{5}\right)$ subcutaneously in the right hind limb. PD-1 inhibition therapy was performed with $200 \mu \mathrm{g} /$ mouse anti-PD-1 antibody (clone RMP1-14, BioXCell) and compared against matched isotype (clone 2A3, BioXCell). For L-1 treatment experiments, L-1 was dissolved in DMSO and diluted to $10 \%$ (v/v) DMSO and 5\% (v/v) cremophor-EL (Sigma-Aldrich) in PBS to be administered intraperitoneally at $10 \mathrm{mg} / \mathrm{kg} / \mathrm{dose}$. Alternatively, L-1 was administered via subcutaneously implanted osmotic pumps filled with L-1 in DMSO/PBS/cremophor-EL formulation with the volume ratio of 2:2:1 in Alzet pumps (catalog 2002 or 1007D) appropriate for the duration of the experiment. Vehicle injections contained 10\% DMSO and 5\% cremophor-EL in PBS. In vivo depletion antibodies were purchased from BioXCell, diluted in sterile PBS, and were administered intraperitoneally at $200 \mu \mathrm{g}$ per mouse twice weekly (anti-CD8, clone 2.43; anti-CD4, clone GK1.5; anti-NK1.1, clone PK136; anti-F4/80, clone Cl:A3-1).

Human peripheral blood samples. Baseline samples from 4 deidentified patients with hepatocellular carcinoma treated at the Sidney Kimmel Comprehensive Cancer Center at Johns Hopkins were identified from the Johns Hopkins Liver Cancer biobank. Blood was collected in 2 BD Vacutainer CPT - Cell Preparation Tube with Sodium Heparin (BD Biosciences) and processed within 2 hours of collection. The tubes were centrifuged at room temperature for approximately 30 minutes at $1800 \mathrm{~g}$. PBMCs were aspirated and pooled into a separate $50 \mathrm{~mL}$ conical tube and washed with RPMI medium. PBMCs were then counted and resuspended in AIM5 and 10\% DMSO at a concentration of $5 \times 10^{6}$ cells per vial. Cryovials were initially stored at $-80^{\circ} \mathrm{C}$ and transferred to liquid nitrogen for long-term storage prior to staining.

PheWAS and BioVU database analyses. A description and methods related to the BioVU database are provided on this website: https:// victr.vumc.org/biovu-description/. The allele frequencies and association analysis of rs2476601 in the PheWAS_GWAS table were calculated using MEGA (Multi-Ethnic Genotyping Array) data. For casecontrolled analysis to determine the association of the PTPN22 SNP variant rs2476601 with response to checkpoint immunotherapy, a total of 137 patients were evaluated between the SNP variant arm $(n=68$ patients) and the control ( $n=69$ patients). Progression-free survival was compared with Kaplan-Meier curves by the log-rank test. Detailed methods are provided in the supplemental material.

TCGA. RNA sequencing (RNA-seq) level 3 RSEM-normalized data from TCGA were accessed from the Broad Institute TCGA GDAC Firehose (http://ezid.cdlib.org/id/doi:10.7908/C16W9975; accessed October, 2018 through March, 2019) and $\log _{2}$ transformed. As a measure of mutational burden, we used the $\log _{2}$-transformed number of nonsynonymous mutations per sample from the TCGA MC3 project (39). CIBERSORT scores for the TCGA samples were accessed from 


\section{Table 2. Adverse events}

\begin{tabular}{|c|c|c|c|}
\hline Event & $\begin{array}{l}\text { All patients } \\
(n=137)\end{array}$ & $\begin{array}{l}\text { rs2476601 SNP } \\
\quad(n=68)\end{array}$ & $\begin{array}{c}\text { Control group } \\
(n=69)\end{array}$ \\
\hline Patients with $\geq 1$ adverse events noted during treatment, no. (\%) & $101(75)$ & $52(77)$ & $49(71)$ \\
\hline Patients $\geq 2$ adverse events noted during treatment, no. (\%) & $66(48)$ & $33(49)$ & $30(44)$ \\
\hline \multicolumn{4}{|l|}{ Most common adverse events noted during treatment, no. } \\
\hline Colitis & 10 & 6 & 4 \\
\hline Diarrhea & 5 & 3 & 2 \\
\hline Dyspena & 11 & 7 & 4 \\
\hline Electrolyte imbalance (Hypocalcemia,hypokalemia,hyponatremia) & 6 & 1 & 5 \\
\hline Fatigue & 22 & 11 & 11 \\
\hline Fever & 6 & 3 & 3 \\
\hline Hepatitis & 6 & 2 & 4 \\
\hline Hypophysitis & 8 & 5 & 3 \\
\hline Hypothyroidism & 10 & 6 & 4 \\
\hline Pain & 8 & 5 & 3 \\
\hline Pneumonitis & 7 & 3 & 4 \\
\hline Rash & 10 & 5 & 5 \\
\hline Weight loss & 6 & 5 & 1 \\
\hline Adverse event resulting in discontinuation of treatment, no. (\%) & $30(22)$ & $18(26)$ & $12(17)$ \\
\hline Colitis & $g^{A}$ & $6^{A}$ & 3 \\
\hline COPD & 2 & 2 & 0 \\
\hline Diabetes mellitus & 1 & 1 & 0 \\
\hline Dyspena & 1 & 1 & 0 \\
\hline Gastritis & 1 & 1 & 0 \\
\hline Hepatitis & 4 & 2 & 2 \\
\hline Hypophisitis & $2^{A}$ & 1 & $1^{A}$ \\
\hline Hypopituitarism & 1 & 0 & 1 \\
\hline Limbic encephalitis & 1 & 1 & 0 \\
\hline Mucositis & 1 & 1 & 0 \\
\hline Neutropenia & 1 & 1 & 0 \\
\hline Pneumonitis & $4^{\mathrm{A}}$ & 1 & $3^{A}$ \\
\hline Rash & 1 & 0 & 1 \\
\hline Tachycardia & 1 & 0 & 1 \\
\hline
\end{tabular}

${ }^{A}$ One case in which therapy was resumed after adverse event was under control.

samples with anti-CD45 antibodies (for immune cells) with or without antiCD29 and anti-CD98 antibodies (for nonimmune cells) conjugated to unique metal tags (43). After 2 washes in cell staining buffer (CSB) (Fluidigm), samples were multiplexed. Samples were first stained with Fc block $(2 \mu \mathrm{g} / 1-3 \times$ $10^{6}$ cells, BD Biosciences) for 10 minutes at room temperature, the surface stain cocktail for 30 minutes at room temperature, washed twice in CSB, and then fixed/permeabilized using a Foxp3 staining kit (eBiosciences) according to the manufacturer's instructions. Conjugation of all custom antibodies was performed as previously described (43). For phospho-profiling assays, cells were plated in 96-well plates and allowed to rest in serum-free RPMI for 2 hours at $37^{\circ} \mathrm{C}$ in $5 \% \mathrm{CO}_{2}$. Cells were moved to ice for incubation with $3.1 \mathrm{mM} \mathrm{H}_{2} \mathrm{O}_{2}$ for 5 minutes at room temperature or anti-CD3 $(1 \mu \mathrm{g} / 100 \mu \mathrm{L} ; 145-2 \mathrm{C} 11$ for hamster anti-mouse; OKT3 for mouse anti-human) for 15 minutes on ice followed by cross-linking secondary antibodies (goat anti-mouse IgG 1 $\mu \mathrm{g} / 100 \mu \mathrm{L}$, Thermo Fisher Scientific; mouse anti-hamster IgG $0.5 \mu \mathrm{g} / 100$ $\mu \mathrm{L}, \mathrm{BD}$ Biosciences) for 15 minutes on ice. Cells were then incubated at $37^{\circ} \mathrm{C}$ for 5 minutes and fixed immediately in $1.6 \%$ paraformaldehyde (PFA) (Thermo Fisher Scientific). After centrifugation, cells were washed twice with CSB, Fc blocked (as detailed above for mouse samples; Purified Human Fc Receptor

Thorsson et al. (40), and xCell scores were downloaded from the xCell web site (https://xcell.ucsf.edu/; accessed October, 2018 through March, 2019) (18). Spearman's correlation coefficients are reported for correlation of Ptpn22 expression and CIBERSORT or xCell scores and mutational burden. Pearson's correlation coefficients are reported for correlation of expression of Ptpn22 and immune regulation markers. Univariate survival analyses were generated from The Cancer Immunome Atlas (https://tcia.at/home) (41).

PTPN22 small molecule inhibitor design, synthesis, and characterization. All compound synthesis and characterization methods are provided in the supplemental material.

Antibodies. All antibodies used for CyTOF and flow cytometry experiments are listed in Supplemental Tables 2-7.

CyTOF staining and data analysis. All CyTOF staining and analyses were performed as previously reported (42). Briefly, $1.5 \times 10^{6}$ cells from each tumor were plated in 96-well plates and washed once in PBS with 2 mM EDTA. For all immune profiling assays, cells were incubated for 5 minutes at room temperature in $500 \mathrm{nM}^{104-110} \mathrm{Pd}$ (Sigma-Aldrich) in PBS for viability staining. Barcoding was performed by incubating
Binding Inhibitor for human samples, eBioscience) for 10 minutes at room temperature, stained for the surface markers for 30 minutes at room temperature, washed again with CSB, and permeabilized with $4^{\circ} \mathrm{C}$ methanol for 10 minutes on ice. Samples were again washed twice with CSB. For either application, the fixed/permeabilized samples were stained using the intracellular cocktail stain for 30 minutes at room temperature. After 2 additional washes, cells were stored in $1.6 \%$ fresh PFA in PBS until the day of acquisition up to 7 days. On the day of data acquisition, samples were stained with 1:1000 ${ }^{103} \mathrm{Rh}$ in Maxpar Fix/ Perm solution (Fluidigm) for 30 minutes at room temperature for cell identification. After washing the samples in CSB, samples were washed with filtered double-distilled water and resuspended in normalization beads (EQ Beads, Fluidigm). All mass cytometry data were acquired at the University of Maryland School of Medicine Center for Innovative Biomedical Resources (CIBR) Flow Cytometry and Mass Cytometry Core Facility, Baltimore, Maryland or Johns Hopkins Mass Cytometry Facility. All acquired data were randomized and normalized using CyTOF software (v6.7, Fluidigm) and debarcoded by manual gating using FlowJo (v10.5, BD). Dead cells were removed by manual gating 
of cells doubly positive for ${ }^{106} \mathrm{Pd}$ and ${ }^{108} \mathrm{Pd}$. Each processed sample was exported as individual fcs files. For clustering analysis, a modified pipeline from Nowicka et al. (44) was used in R (v3.5), employing FlowSOM (21) and UMAP (22) algorithms.

Flow cytometry. For each sample, $1.5 \times 10^{6}$ cells were plated in 96-well plates and washed with PBS containing $2 \mathrm{mM}$ EDTA. Cells were Fc blocked for 10 minutes at $4^{\circ} \mathrm{C}$, after which the samples were incubated in the surface staining cocktail for 30 minutes at $4^{\circ} \mathrm{C}$. For tetramer analysis, cells were first stained with $\mathrm{H}-2 \mathrm{~K}^{\mathrm{b}}$ SIINFEKL tetramers $(\mathrm{NIH}$ Tetramer Core) for 45 minutes at room temperature. After washing 3 times with CSB, cells were stained with anti-CD8 antibody (1:100, clone $\mathrm{KT} 15, \mathrm{MBL}$ International) for 30 minutes at $4^{\circ} \mathrm{C}$. For phospho-flow validation of clones and staining, the same stimulation and staining protocol detailed for mass cytometry was used. For transcription factors, cells were fixed/permeabilized using a Foxp3 staining kit and stained intracellularly for 45 minutes at room temperature. Before data acquisition, cells were washed twice in CSB, and data were collected on a CytoFLEX (Beckman). The gating strategy used to identify tumor-infiltrating T cells is shown in the supplemental material. For coculture experiments, cells were stained with a cocktail including Fc block (2.4G2) and antibodies against the following proteins for 30 minutes at room temperature: CD3, CD4, CD8a, CD44, and CD69. Cells were fixed and permeabilized using a Cytofix/Cytoperm solution (BD Biosciences) for 30 minutes at room temperature. After a series of washes, cells were then stained with an intracellular cocktail consisting of antibodies against the following proteins for 30 minutes at room temperature: granzyme B, IFN- $\gamma$, and IL-2. All data were collected on a BD CytoFLEX flow cytometer.

Immunohistochemistry. All samples were formalin fixed and paraffin embedded (FFPE) and slides were prepared according to standard histological procedures. Immunohistochemistry for CD4, CD8, and Foxp3 was performed at the Johns Hopkins Oncology Tissue Services on a Ventana Discovery Ultra autostainer (Roche Diagnostics). Briefly, slides were dewaxed and rehydrated, and epitope retrieval was performed using Ventana Ultra CC1 buffer (Roche Diagnostics) at $96^{\circ} \mathrm{C}$ for 64 minutes. Primary antibody for CD4 (1:1000, catalog ab183685, Abcam), CD8 (1:125, clone 4SM16, Thermo Fisher Scientific), or Foxp3 (1:75, clone D6O8R, Cell Signaling Technologies) was applied at $36^{\circ} \mathrm{C}$ for 60 minutes, followed by application of rabbit anti-rat linker antibody (1:500, Vector Labs) at $36^{\circ} \mathrm{C}$ for 32 minutes, anti-rabbit HQ detection system, or anti-rabbit HQ detection system with Discovery AMP Multimer (Roche Diagnostics), respectively. Chromomap DAB IHC detection kit (Roche Diagnostics) was used. Counterstaining was done with Mayer's hematoxylin. Slides were dehydrated and mounted with coverslips.

TCR sequencing. Harvested MC38 tumors were stored as FFPE samples and subsequently cut into scrolls for sequencing using the immunoSeq assay (Adaptive) at survey depth. Shannon's entropy and clonality were quantified as previously described (45).

Coculture experiments. Tumors from mice inoculated with MC38OVA cells were harvested and enzymatically dissociated into single cells and enriched for $\mathrm{F} 4 / 80^{+}$cells using a positive selection kit (Miltenyi Biotec). $\mathrm{F} 4 / 80^{+}$cells were then plated at 100,000 cells per well on a $96-$-well plate. Spleens were harvested from OT-I and OT-II mice and dissociated into single cells through a $40 \mu \mathrm{m}$ strainer. These cells were then enriched for $\mathrm{CD}^{+} \mathrm{T}$ cells (from the OT-I splenocytes) and $\mathrm{CD} 4^{+} \mathrm{T}$ cells (from the OT-II splenocytes) using a negative selection kit (Miltenyi Biotec). CD8 ${ }^{+}$ T cells and $\mathrm{CD}^{+}{ }^{+} \mathrm{T}$ cells were then stained with CFSE (Thermo Fisher Scientific) at a final concentration of $80 \mu \mathrm{M}$. Following CFSE staining, CD ${ }^{+}$
T cells and $\mathrm{CD} 4^{+} \mathrm{T}$ cells were added to the wells containing $\mathrm{F} 4 / 80^{+}$cells at 2 different concentrations, either $0.2 \times 10^{6}$ or $1 \times 10^{6}$ cells per well. Control wells were also included using $1 \times 10^{6}$ cells per well of $\mathrm{CD} 4^{+} \mathrm{T}$ cells and $\mathrm{CD}^{+} \mathrm{T}$ cells cocultured with mouse T-activator CD3/CD28 Dynabeads (Invitrogen). All wells included $50 \mathrm{ng} / \mathrm{mL} \mathrm{IL-2}$ and were incubated at $37^{\circ} \mathrm{C}$ and $5 \% \mathrm{CO}_{2}$ for 72 hours. In the final 2.5 hours of this incubation, a brefeldin A solution (BioLegend) was added at a concentration of $5 \mu \mathrm{g} /$ $\mathrm{mL}$ to each well. Following the final 2.5-hour incubation, the Dynabeads were removed magnetically prior to staining for flow cytometry.

Statistics. For differential analysis of the CyTOF data sets, linear mixed modeling was used. Unless otherwise noted, an unpaired 2-tailed $t$ test was used to test differences between 2 groups and 1-way ANOVA for more than 2 groups. A $P$ value of less than 0.05 was considered significant. Statistical analysis for significant differences in the tumor growth curves were conducted using regression analyses based on nonlinear regression (exponential growth curves). Univariate survival analyses were performed with log-rank tests.

Study approval. All mouse husbandry, experiments, and euthanasia were performed in accordance with Johns Hopkins IACUC-approved protocols. The evaluation of banked human PBMCs was performed in accordance with the protocols approved by the Johns Hopkins Institutional Review Board (IRB). All peripheral blood specimens were obtained with written patient consent (IRB number CIR00051274).

\section{Author contributions}

WJH conceived the study, designed and performed experiments, and analyzed data. WJH and ZHP designed and performed phospho-profiling analysis. MY provided clinical samples for phospho-profiling. J Lin, J Dong, DA, YB, and ZYZ designed, synthesized, and characterized PTPN22 inhibitors, performed pharmacokinetic experiments, and analyzed data. ZHP, S Charmsaz, AAM, NEG, JML, and FC performed animal experiments. TDA analyzed animal experiment data. NZ designed vaccination experiments. WJH and EJF analyzed mass cytometry data sets. LD and EJF analyzed TCGA data sets. S Croessmann, JW, JH, JCD, and BHP analyzed PheWAS/ BioVU data sets. WJH, S Croessmann, and J Lin are co-first authors based on their distinct contributions; the order of co-first authors was determined based on the overall scientific contribution. BHP and EMJ designed experiments and analyzed data. WJH, S Croessmann, and J Lin wrote the manuscript. BHP, ZYZ, and EMJ provided funding. All authors revised the manuscript.

\section{Acknowledgments}

The results here are in whole or part based on data generated by TCGA Research Network. The authors thank Sanford Markowitz for critical discussions. WJH is the recipient of the American Society of Clinical Oncology Young Investigator Award, American Association of Cancer Research Incyte Immuno-Oncology Research Fellowship, MacMillan Pathway to Independence Award, and was supported by NIH grant T32CA00971-38. JL, JD, DA, YB, and ZYZ are supported by NIH grant RO1 CA207288. JCD, JW, and JH are supported by NIH grant R01 LM010685. This work was also supported by the Breast Cancer Research Foundation, the Komen Foundation, and NIH grants CA214494 and CA194024 (to BHP). We would also like to thank and acknowledge the support of the Canney Foundation, the Steve Kandel Foundation, Amy and Barry Baker, a VanderbiltIngram Cancer Center support grant (NIH grant CA068485), and a 
Breast Cancer SPORE grant (NIH grant CA098131). JCD's involvement in this project was primarily as faculty at Vanderbilt University Medical Center prior to joining the NIH. The Vanderbilt University Medical Center's BioVU data sets were supported by numerous sources: institutional funding, private agencies, and federal grants. These include the NIH-funded Shared Instrumentation Grant S10RR025141; and CTSA grants UL1TR002243, UL1TR000445, and UL1RR024975. BioRender was used to generate schematics.
Address correspondence to: Ben Ho Park, 777 Preston Research Building, 2220 Pierce Avenue, Nashville, Tennessee 37232, USA. Email: ben.h.park@vumc.org. Or to: Zhong-Yin Zhang, 575 Stadium Mall Drive, RHPH 202A, West Lafayette, Indiana 47907, USA. Phone: 765.496.3720; Email: zhang-zy@purdue.edu. Or to: Elizabeth Jaffee, 4M07 Bunting Blaustein Cancer Research Building, 1650 Orleans Street, Baltimore, Maryland, 21287, USA. Phone: 410.955.2957; Email: ejaffee@jhmi.edu.
1. Rosenberg SA. Decade in review-cancer immunotherapy: entering the mainstream of cancer treatment. Nat Rev Clin Oncol. 2014;11(11):630-632.

2. Fesnak AD, et al. Engineered T cells: the promise and challenges of cancer immunotherapy. Nat Rev Cancer. 2016;16(9):566-581.

3. Pardoll DM. The blockade of immune checkpoints in cancer immunotherapy. Nat Rev Cancer. 2012;12(4):252-264.

4. Ribas A, Wolchok JD. Cancer immunotherapy using checkpoint blockade. Science. 2018;359(6382):1350-1355

5. Bottini N, Peterson EJ. Tyrosine phosphatase PTPN22: multifunctional regulator of immune signaling, development, and disease. Annu Rev Immunol. 2014;32:83-119.

6. Stanford SM, Bottini N. PTPN22: the archetypal non-HLA autoimmunity gene. Nat Rev Rheumatol. 2014;10(10):602-611.

7. Wu J, et al. Identification of substrates of human protein-tyrosine phosphatase PTPN22. J Biol Chem. 2006;281(16):11002-11010.

8. Begovich $\mathrm{AB}$, et al. A missense single-nucleotide polymorphism in a gene encoding a protein tyrosine phosphatase (PTPN22) is associated with rheumatoid arthritis. Am J Hum Genet. 2004;75(2):330-337.

9. Bottini $\mathrm{N}$, et al. A functional variant of lymphoid tyrosine phosphatase is associated with type I diabetes. Nat Genet. 2004;36(4):337-338.

10. Kyogoku C, et al. Genetic association of the R620W polymorphism of protein tyrosine phosphatase PTPN22 with human SLE. Am J Hum Genet. 2004;75(3):504-507.

11. Dai X, et al. A disease-associated PTPN22 variant promotes systemic autoimmunity in murine models. J Clin Invest. 2013;123(5):2024-2036.

12. Hasegawa K, et al. PEST domain-enriched tyrosine phosphatase (PEP) regulation of effector/memory T cells. Science. 2004;303(5658):685-689.

13. Brownlie RJ, et al. Resistance to TGF $\beta$ suppression and improved anti-tumor responses in $\mathrm{CD}^{+} \mathrm{T}$ cells lacking PTPN22. Nat Commun. 2017;8(1):1343.

14. Brownlie RJ, et al. Deletion of PTPN22 improves effector and memory CD8 ${ }^{+} \mathrm{T}$ cell responses to tumors. JCI Insight. 2019;5:e127847.

15. Cubas R, et al. Autoimmunity linked protein phosphatase PTPN22 as a target for cancer immunotherapy. JImmunother Cancer. 2020;8(2):e001439.

16. Orozco RC, et al. Pro-autoimmune allele of tyrosine phosphatase, PTPN22, enhances tumor immunity [preprint]. https://doi. org/10.1101/2021.03.17.435898. Posted on bioRxiv March 18, 2021.

17. Denny JC, et al. Systematic comparison of phenome-wide association study of electronic medical record data and genomewide association study data. Nat Biotechnol. 2013;31(12):1102-1110.

18. Aran D, et al. xCell: digitally portraying the tissue cellular heterogeneity landscape. Genome Biol. 2017;18(1):220.

19. Newman AM, et al. Robust enumeration of cell subsets from tissue expression profiles. Nat Methods. 2015;12(5):453-457.

20. Bendall SC, et al. Single-cell mass cytometry of differential immune and drug responses across a human hematopoietic continuum. Science. 2011;332(6030):687-696.

21. Van Gassen S, et al. FlowSOM: Using self-organizing maps for visualization and interpretation of cytometry data. Cytometry Part A. 2015;87(7):636-645.

22. Becht E, et al. Dimensionality reduction for visualizing single-cell data using UMAP. Nat Biotechnol. 2019;37:38-47.

23. Karandikar SH, et al. New epitopes in ovalbumin provide insights for cancer neoepitopes. JCI Insight. 2019;5:e127882.

24. Karan D, et al. Paradoxical enhancement of CD8 $\mathrm{T}$ cell-dependent anti-tumor protection despite reduced CD8 $\mathrm{T}$ cell responses with addition of a TLR9 agonist to a tumor vaccine. Int J Cancer. 2007;121(7):1520-1528.

25. Salvador JM, et al. The autoimmune suppressor Gadd45alpha inhibits the T cell alternative p38 activation pathway. Nat Immunol. 2005;6(4):396-402.

26. Salvador JM, et al. Alternative p38 activation pathway mediated by $\mathrm{T}$ cell receptor-proximal tyrosine kinases. Nat Immunol. 2005;6(4):390-395

27. Pauken KE, Wherry EJ. Overcoming T cell exhaustion in infection and cancer. Trends Immunol. 2015;36(4):265-276.

28. Wherry EJ, Kurachi M. Molecular and cellular insights into T cell exhaustion. Nat Rev Immunol. 2015;15(8):486-499.

29. Wang LX, et al. M2b macrophage polarization and its roles in diseases. J Leukoc Biol. 2019;106(2):345-358.

30. Blackburn SD, et al. Selective expansion of a subset of exhausted CD8 T cells by alphaPD-L1 blockade. Proc Natl Acad Sci U S A. 2008;105(39):15016-15021.

31. Ahn E, et al. Role of PD-1 during effector CD8 T cell differentiation. Proc Natl Acad Sci US A.
2018;115(18):4749-4754

32. Chang $\mathrm{H}-\mathrm{H}$, et al. PTPN22 modulates macrophage polarization and susceptibility to dextran sulfate sodium-induced colitis. JImmunol. 2013;191(5):2134-2143.

33. Spalinger MR, et al. Loss of protein tyrosine phosphatase nonreceptor type 22 regulates interferon- $\gamma$-induced signaling in human monocytes. Gastroenterology. 2013;144(5):978-988.

34. Zhang J, et al. The autoimmune disease-associated PTPN22 variant promotes calpain-mediated Lyp/Pep degradation associated with lymphocyte and dendritic cell hyperresponsiveness. Nat Genet. 2011;43(9):902-907.

35. Nathan CF, et al. Identification of interferon-gamma as the lymphokine that activates human macrophage oxidative metabolism and antimicrobial activity. J Exp Med. 1983;158(3):670-689.

36. Chen YNP, et al. Allosteric inhibition of SHP2 phosphatase inhibits cancers driven by receptor tyrosine kinases. Nature. 2016;535(7610):148-152.

37. Hoekstra E, et al. Meeting Report Europhosphatase 2015: phosphatases as drug targets in cancer. Cancer Res. 2016;76(2):193-196.

38. Zhang ZY. Drugging the undruggable: therapeutic potential of targeting protein tyrosine phosphatases. Acc Chem Res. 2017;50(1):122-129.

39. Ellrott K, et al. Scalable open science approach for mutation calling of tumor exomes using multiple genomic pipelines. Cell Syst. 2018;6(3):271-281.

40. Thorsson V, et al. The immune landscape of cancer. Immunity. 2018;48(4):812-830.

41. Charoentong $\mathrm{P}$, et al. Pan-cancer immunogenomic analyses reveal genotype-immunophenotype relationships and predictors of response to checkpoint blockade. Cell Rep. 2017;18(1):248-262.

42. Ho WJ, et al. Multi-panel mass cytometry reveals anti-PD1 therapy-mediated B and T cell compartment remodeling in tumor-draining lymph nodes. JCI Insight. 2019;5(2):e132286.

43. Charmsaz S, et al. A global live cell barcoding approach for multiplexed mass cytometry profiling of mouse tumors. JCI Insight. 2021;6(7):e143283.

44. Nowicka M, et al. CyTOF workflow: differential discovery in high-throughput high-dimensional cytometry datasets. F1000Res. 2019;6:748.

45. Hopkins AC, et al. T cell receptor repertoire features associated with survival in immunotherapy-treated pancreatic ductal adenocarcinoma. JCI Insight. 2018;3(13):e122092. 